\title{
RESEARCH
}

Open Access

\section{MicroRNA-153 impairs presynaptic plasticity by blocking vesicle release following chronic brain hypoperfusion}

Mei-Ling Yan ${ }^{\dagger}$, Shuai Zhang ${ }^{\dagger}$, Hong-Mei Zhao, Sheng-Nan Xia, Zhuo Jin, Yi Xu, Lin Yang, Yang Qu, Si-Yu Huang, Ming-Jing Duan, Meng Mao, Xiao-Bin An, Chandan Mishra, Xin-Yu Zhang, Li-Hua Sun and Jing Ai

\begin{abstract}
Background: Chronic brain hypoperfusion (CBH) is closely related to Alzheimer's disease (AD) and vascular dementia (VaD). Meanwhile, synaptic pathology plays a prominent role in the initial stage of AD and VaD. However, whether and how $\mathrm{CBH}$ impairs presynaptic plasticity is currently unclear.

Methods: In the present study, we performed a battery of techniques, including primary neuronal culture, patch clamp, stereotaxic injection of the lentiviral vectors, morris water maze (MWM), dual luciferase reporter assay, FM143 fluorescence dye evaluation, qRT-PCR and western blot, to investigate the regulatory effect of miR-153 on hippocampal synaptic vesicle release both in vivo and in vitro. The CBH rat model was generated by bilateral common carotid artery ligation (2VO).

Results: Compared to sham rats, 2VO rats presented decreased field excitatory postsynaptic potential (fEPSP) amplitude and increased paired-pulse ratios (PPRs) in the CA3-CA1 pathway, as well as significantly decreased expression of multiple vesicle fusion-related proteins, including SNAP-25, VAMP-2, syntaxin-1A and synaptotagmin-1, in the hippocampi. The levels of microRNA-153 (miR-153) were upregulated in the hippocampi of rats following $2 \mathrm{VO}$ surgery, and in the plasma of dementia patients. The expression of the vesicle fusion-related proteins affected by 2VO was inhibited by miR-153, elevated by miR-153 inhibition, and unchanged by binding-site mutation or miR masks. FM1-43 fluorescence images showed that miR-153 blunted vesicle exocytosis, but this effect was prevented by either 2'-O-methyl antisense oligoribonucleotides to miR-153 (AMO-153) and miR-masking of the miR-153 binding site in the 3' untranslated region (3'UTR) of the Snap25, Vamp2, Stx1a and Syt1 genes. Overexpression of miR-153 by lentiviral vector-mediated miR-153 mimics (lenti-pre-miR-153) decreased the fEPSP amplitude and elevated the PPR in the rat hippocampus, whereas overexpression of the antisense molecule (lenti-AMO-153) reversed these changes triggered by $2 \mathrm{VO}$. Furthermore, lenti-AMO-153 attenuated the cognitive decline of 2VO rats. (Continued on next page)
\end{abstract}

\footnotetext{
* Correspondence: azhrbmu@126.com; aijing@ems.hrbmu.edu.cn

${ }^{\dagger}$ Mei-Ling Yan and Shuai Zhang contributed equally to this work.

Department of Pharmacology (The State-Province Key Laboratories of Biomedicine-Pharmaceutics of China), College of Pharmacy of Harbin Medical University, Harbin 150086, Heilongjiang Province, China
} 


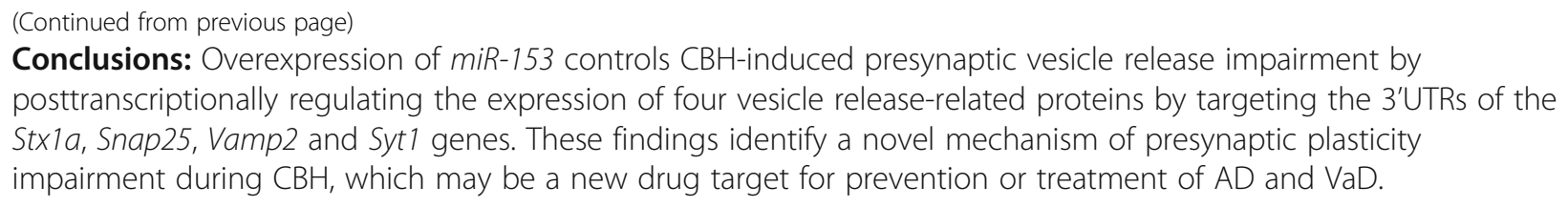

Keywords: Chronic brain hypoperfusion, Presynaptic plasticity, microRNA-153, Synaptic vesicle fusion, Low cerebral blood flow

\section{Background}

Recently, chronic brain hypoperfusion $(\mathrm{CBH})$ has been considered serving as a key predictor of the conversion of mild cognitive impairment (MCI) to Alzheimer's disease (AD) and vascular dementia $(\mathrm{VaD})$ [1]. Previous studies have documented that $\mathrm{CBH}$ can produce cognitive decline by affecting many pathological processes including white matter attenuation, neuronal death, neuroinflammation, oxidative stress and even the formation of $A \beta$ and the hyperphosphorylation of Tau [1-6]. Synaptic pathology plays a prominent role in the initial stage of $\mathrm{AD}$ and $\mathrm{VaD}$ [7-9]. CBH also attenuates synaptic plasticity by changing the expression of several proteins and remodeling synaptic dendrites and spines $[10,11]$, however, whether and how CBH impairs presynaptic plasticity is currently unclear.

Presynaptic plasticity in chemical synapses is based on neurotransmitter release through synaptic vesicle exocytosis, which relies on highly conserved protein machinery. Neurotransmitter release is well understood to be dependent on the fusion of presynaptic vesicles with presynaptic membranes. This process is controlled by the interaction between the $\mathrm{Ca}^{2+}$ sensor protein synaptotagmin-1 (Syt1) and the soluble $N$-ethylmaleimide-sensitive factor attachment receptor proteins complex (SNAREs), which comprises vesicle associated membrane protein 2 (VAMP-2), synaptosomal-associated protein 25 (SNAP-25) and syntaxin-1 [12, 13]. Other important proteins that are required for exocytosis, including Munc-18, Munc-13 and complexins, have been found to collaborate with SNAREs to enhance the fusion probability between vesicles and presynaptic membranes [14, 15]. Importantly, the expression of SNAP-25, VAMP-2, syntaxin-1 and Syt1 has been reported to be lower in postmortem tissues from $\mathrm{AD}$ and $\mathrm{VaD}$ patients than in those from healthy controls [16-19]. Whether CBH directly affects presynaptic plasticity by interrupting this highly conserved and elaborate protein machinery is unclear.

Previous studies have reported that microRNAs (miRNAs) could regulate presynaptic plasticity by targeting various vesicle-related proteins. For example, miR-137 impaired vesicle release in a mouse model of schizophrenia [20]; miR-34c was reported mediate amyloid-beta $(\mathrm{A} \beta)$-induced synaptic failure by targeting VAMP2 [21]; knockdown of miR-135a triggered an early stress response by disturbing presynaptic vesicle release [22]; miR-153 was found to be a contextual fear-induced miRNA in the dentate gyrus [23] and was reported to regulate vesicle release in zebrafish [24]. However, these studies did not analyze $\mathrm{CBH}$-induced changes in miRNA levels or their function in presynaptic plasticity.

Here we show that $\mathrm{CBH}$ obstructs presynaptic vesicle fusion with presynaptic membrane via miR-153-mediated downregulation of multiple synaptic vesicle-related proteins. Therefore, we consider that $m i R-153$ may be a new drug target for the treatment of dementia.

\section{Methods \\ Animals}

Male Sprague-Dawley (SD) rats ( $5 \sim 6$ months old) were obtained from the Animal Center of the Second Affiliated Hospital of Harbin Medical University (Harbin, Heilongjiang Province, China). All animals for experiments were housed at $23 \pm 1{ }^{\circ} \mathrm{C}$ with $55 \pm 5 \%$ humidity and maintained on a 12-h dark-light artificial cycle (lights on at 7:00 AM) with food and water available ad libitum. Samples for quantitative real-time PCR (qRTPCR) and western blot assay were obtained from the hippocampi and/or cortices of rats after they had been anaesthetized with $10 \%$ chloral hydrate $(500 \mathrm{mg} / \mathrm{kg}$, intraperitoneal, Aladdin, Cat. No. C104202, Shanghai, China) followed by confirmation of death by exsanguination. Tissues for primary neuron culture were obtained from neonatal SD rats after administration of $20 \%$ isoflurane and confirmation of death by cervical dislocation. All animal procedures were approved by the Institutional Animal Care and Use Committee at Harbin Medical University (No. HMUIRB-2008-06) and the Institute of Laboratory Animal Science of China (A5655-01). All procedures were conformed to the Directive 2010/63/EU of the European Parliament.

\section{Construction of lentivirus vectors}

Lenti-pre-miR-153, the miR-153-3p overexpression plasmid (RmiR6060-MR03, GeneCopoeia, Guangzhou, China) or negative control (NC) using scrambled RNA oligonucleotides of miR-153 (lenti-mis-pre-miR-153-3p) (CmiR0001-MR03, GeneCopoeia, Guangzhou, China) were generated using the pEZX-MR03 lentiviral transfer 
vector; while, the miR-153 inhibitor plasmid lenti-AMO153-3p or NC of AMO-153, using scrambled RNA oligonucleotides (leni-mis-AMO-153-3p), was generated using pEZX-AM03 (RmiR-AN0214-AM03, GeneCopoeia, Guangzhou, China). The titers of these RNA oligonucleotide vectors used for experiments were $1 \times$ $10^{8}$ transducing $\mathrm{U} / \mathrm{mL}$.

\section{Permanent, bilateral common carotid artery occlusion (2VO) in the rat}

The method used for preparation of $2 \mathrm{VO}$ rats has been describedin previous studies [3, 25] (www.bio-protocol. org/e2668). Briefly, after being anaesthetized by $10 \%$ chloral hydrate $(300 \mathrm{mg} / \mathrm{kg})$ and maintained under anaesthesia using $0.5-1.0 \%$ isoflurane, rats were placed on an electric heating pad (Dongxiyi, Cat. No. wi95919, Beijing, China) to maintain body temperature at $37^{\circ} \mathrm{C}$. Then the fur around the neck was removed using an electric shaver and the area was sterilized by a $75 \%$ alcohol cotton ball. A 2-cm incision was made above the manubrium along the anterior midline of the neck. The bilateral common carotid arteries of rats were exposed by vertical separation of the omohyoideus muscle and then carefully separated from the vagus and aortic depressor nerve followed by permanent ligation with 3-0 silk suture (Jinhuan, model: $3-0$, China). After the surgical procedures, all the anterior cervical muscles were returned to their original location. To avoid potential postoperative infection, the wounds were washed before closing with $20 \mathrm{mg} / \mathrm{mL}$ gentamycin sulfate solution (Sangon Biotech, Cat. No. B540724). The wounds were then sutured and rats were allowed to recover from anaesthesia before being returned back to their cages.

\section{Stereotaxic injection of the lentiviral vectors}

After anaesthesia, rats were placed on a stereotaxic frame (RWB Life Science Co. Ltd., China), and the skull was exposed. Then, $2 \mu \mathrm{L}\left(10^{8} \mathrm{TU} / \mathrm{mL}\right)$ lenti-pre-miR153-3p, lenti-2'-O-methyl antisense oligoribonucleotides to $m i R-153$ (AMO-153), lenti-mis-pre-miR-153-3p and/ or leni-mis-AMO-153-3p was injected into the bilateral CA1 region of the hippocampus using a $5-\mu \mathrm{L}$ Hamilton syringe with a 33-gauge tip needle (Hamilton, Bonaduz, Switzerland) at a rate of $0.5 \mu \mathrm{L} / \mathrm{min}$. The injection coordinate relative to the bregma was as follows: anteroposterior (AP), $-3.60 \mathrm{~mm}$; mediolateral (ML), \pm 2.30 $\mathrm{mm}$; dorsoventral (DV), $-3.00 \mathrm{~mm}$ below the surface of the dura using coordinates derived from the atlas of Paxinos and Watson. The needle was maintained in the place for another $2 \mathrm{~min}$ after injection and then withdrawn very slowly to prevent backflow of the solution. Finally, the skin incision was sutured, and the animal was returned to its housing after recovering from anaesthesia. Subsequent experiments were performed 8 weeks after virus injection [3].

\section{Morris water maze (MWM)}

The maze consisted of a black circular pool of $2.0 \mathrm{~m}$ diameter, filled with opaque water $\left(25 \pm 1{ }^{\circ} \mathrm{C}\right)$ via the addition of black food pigment. A submerged escape platform $(20 \mathrm{~cm}$ in diameter, top surface $2.0 \mathrm{~cm}$ below water level) was located in the centre of the first quadrant. Before training, the pupillary light reflex of all rats was tested, and those with impaired pupillary light reflex were excluded from the experiment to avoid the influence of the animal's vision on the test. For cued training (three trials per day for $5 \mathrm{~d}$ ), the rats were released into the water facing the sidewalls, and each rat was allowed $120 \mathrm{~s}$ to find the platform, if the rats did not find it in the time allowed, they were guided to the platform and permitted to rest for at least $20 \mathrm{~s}$. After the last cued trial of day 5, the platform was removed from the pool, and each rat was tested on one 120-s swim probe trial on day 6. Escape latency (s) and the number of platform crossings were monitored using an online DigBehavMorris Water Maze Video Analysis System (Mobile Datum Software Technology Co. Ltd., Shanghai, China) $[3,26]$.

\section{Functional magnetic resonance imaging (fMRI) measurements}

After rats were anaesthetized with chloral hydrate (300 $\mathrm{mg} / \mathrm{kg}$,.i.p.), a dose of $0.06 \mathrm{mmoL} / \mathrm{kg}$ of Dimeglumine gadopentetate was injected into the rat's tail vein, and an animal brain coil was used for T2-weighted imaging to acquire fMRI measurements using a 3.0-T animal MIR scanner (PHILIPS: ACHIEVA 3.0-TX) with fast spinecho plus sequences. The parameters were follows: data matrix $=100 \times 92$, repetition time $(\mathrm{TR})=2534 \mathrm{~ms}$, effective echo time $(\mathrm{TE})=40 \mathrm{~ms}$, echo train length $=27$, field of view $=0.8 \times 0.25 \mathrm{~cm}$, twenty-five 1 -mm slices, and four signal averages [27].

\section{2,3,5-Triphenyltetrazolium chloride (TTC) staining}

After the rats were anaesthetized, the brains of rats were immediately removed and cut into $2-\mathrm{mm}$ sections, which were then immersed sequentially into a phosphatebuffered $2 \%$ TTC solution at $37^{\circ} \mathrm{C}$ for $30 \mathrm{~min}$, and then fixed in phosphate-buffered $4 \%$ paraformaldehyde (PFA) solution at $4{ }^{\circ} \mathrm{C}$ [3].

\section{Electrophysiology \\ Hippocampal slice preparation}

The rats were anaesthetized with $20 \%$ urethane intraperitoneally and decapitated. After the skull was exposed, the whole head was removed and carefully placed on ice-cold dissection buffer N-methyl-D-glucamine (NMDG) solution 
in mM: 93 NMDG, $2.5 \mathrm{KCl}, 10 \mathrm{MgSO}_{4}, 1.2 \mathrm{NaH}_{2} \mathrm{PO}_{4}, 30$ $\mathrm{NaHCO}_{3}, 20$ HEPES, $0.5 \mathrm{CaCl}_{2}$, 25 D-glucose, 5 sodiumascorbate, 3 sodium-pyruvate, 2 thiourea and $2 \mathrm{~N}$-acetyl-Lcysteine, with $\mathrm{pH} 7.3-7.4$ adjusted by $\mathrm{NaOH}$ or $\mathrm{HCl}$ and saturated with $\mathrm{O}_{2}(95 \%) / \mathrm{CO}_{2}(5 \%)$ carbogen mixture for $10 \mathrm{~s}$. After the transverse hippocampal slices $(400 \mu \mathrm{m}$ thick) were prepared using a Leica VT1200S microtome (Leica, Nussloch, Germany), the slices were transferred into NMDG solution at $28^{\circ} \mathrm{C}$ for a 3-min recovery, and sequentially maintained in an $\mathrm{O}_{2}(95 \%) / \mathrm{CO}_{2}(5 \%)$-saturated incubation solution containing (in $\mathrm{mM}$ ): $124 \mathrm{NaCl}, 2.5 \mathrm{KCl}, 1.2$ $\mathrm{NaH}_{2} \mathrm{PO}_{4}, 24 \mathrm{NaHCO}_{3}, 2 \mathrm{MgSO}_{4}, 2 \mathrm{CaCl}_{2}, 5$ HEPES, $2 \mathrm{~N}$-acetyl-L-cysteine, and 12.5 D-glucose (pH 7.3-7.4) for at least $1 \mathrm{~h}$ at $33 \pm 2{ }^{\circ} \mathrm{C}$. Then the slices were maintained in standard artificial cerebrospinal fluid (ACSF) solution at a constant flow $(2 \sim 3 \mathrm{~mL} / \mathrm{min})$ and constant temperature of $35 \pm 2{ }^{\circ} \mathrm{C}$ with the help of a temperature controller (TC-324C, Warner Instruments). The ACSF contained the following (in $\mathrm{mM}$ ): $124 \mathrm{NaCl}, 2.5 \mathrm{KCl}, 1.2 \mathrm{NaH}_{2} \mathrm{PO}_{4}, 24 \mathrm{NaHCO}_{3}, 2$ $\mathrm{CaCl}_{2}, 2 \mathrm{MgSO}_{4}, 5$ HEPES and $10 \mathrm{D}$-glucose [28].

\section{Extracellular recordings}

Concentric bipolar microelectrodes (CBARC75, FHC, USA) were placed in the Schaffer collateral (SC) domain of CA3 $300 \mu \mathrm{m}$ away from recording pipettes, which were placed in the stratum radiatum of CA1. Recording pipettes were pulled from borosilicate glass (BF100-5810 , Sutter Instrument) with resistances of $2-3 \mathrm{M} \Omega$ when filled with a $\mathrm{NaCl}(3 \mathrm{~mol} / \mathrm{L})$ solution. The field excitatory postsynaptic potential (fEPSP) of CA3-CA1 was evoked by current stimulation of $0.033 \mathrm{~Hz}$ with stimulation steps of 20 - to $160-\mu \mathrm{A}$ (10- steps, $100 \mathrm{~ms}$ ) using a stimulatory isolator (ISO-Flex (AMPI, Jerusalem, Israel), controlled by a Master- 8 pulse generator (AMPI, Jerusalem, Israel). The stimulus intensities, which corresponded to that necessary to evoke $\sim 50 \%$ of the maximum amplitude of fEPSP, were used for the next synaptic plasticity evaluation [29]. Analogue signals were bypass filtered and digitized at $6 \mathrm{kHz}$ using Digidata 1550A and pClamp10 software (Molecular Devices, US). Paired-pulse facilitation (PPF) in hippocampal CA1 was recorded in separate slices and measured by evoking fEPSP with varying interpulse intervals from 20 to $500 \mathrm{~ms}$.

Signal analysis Off-line analysis was performed using Clampfit software (Molecular Devices, US). The basic synaptic transmission was assessed by the normalized amplitude of fEPSP calculated by the amplitude of each fEPSP relative to the amplitude evoked by $20 \mu \mathrm{A}$ stimulation. Presynaptic vesicle release was evaluated by PPF which is expressed paired-pulse ratio (PPR): fEPSP2 amplitude/fEPSP1 amplitude [30].

\section{Extraction of synaptosomes}

After anaesthetization and perfusion with chilled normal saline, rats were sacrificed with $10 \%$ chloral hydrate $(500 \mathrm{mg} / \mathrm{kg})$, and the hippocampi were quickly removed and immediately placed on an ice-cold plate, followed by suspension in $10 \%(\mathrm{w} / \mathrm{v}) 320 \mathrm{mM}$ sucrose HEPES buffer and homogenization with a grinding rod. The HEPES buffer contained the following (in $\mathrm{mM}$ ): $145 \mathrm{NaCl}, 5$ $\mathrm{KCl}, 2 \mathrm{CaCl}_{2}, 1 \mathrm{MgCl}_{2}, 5$ glucose and 5 HEPEs buffer with $\mathrm{pH}$ 7.3-7.4. Furthermore, the homogenate was centrifuged at $4-8^{\circ} \mathrm{C}$ for $10 \mathrm{~min}$ at $600 \times \mathrm{g}$. The supernatant was then diluted at a 1:1 ratio with $1300 \mathrm{mM}$ sucrose HEPES buffer to yield a suspension at a final concentration of $800 \mathrm{mM}$ sucrose, and centrifuged at $4-8^{\circ} \mathrm{C}$ for $15 \mathrm{~min}$ at $12000 \times \mathrm{g}$. The supernatant was discarded. The pellet consisting of synaptosomes was washed twice with HEPES buffer and centrifuged at $12,000 \times g$ for $15 \mathrm{~min}$ at $4-8{ }^{\circ} \mathrm{C}$ to wash out the impurities [31]. To obtain the synapse proteins for western blot experiments, the resulting synaptosomal preparation was disassociated in RIPA buffer with $0.2 \%$ TritonX-100 and $1 \%$ protease inhibitor. After standing for $30 \mathrm{~min}$ on ice, the suspension was further centrifuged at $20,000 \times g$ for $30 \mathrm{~min}$ at $4-8{ }^{\circ} \mathrm{C}$.

\section{FM1-43 fluorescent dye evaluation}

After the neonatal rat hippocampal and cortical neurons (NRNs) were cultured for 10-14 d in cover glasses, they were incubated with FM 1-43 fluorescent dye (T35356, Invitrogen, Oregon, USA) for $3 \mathrm{~min}$ at room temperature to allow FM1-43 to bind to the outer membrane of NRNs. Next, $70 \mathrm{mM} \mathrm{KCl}$ was added to the FM1-43 loaded NRNs for $3 \mathrm{~min}$ at room temperature to allow for the internalization of FM1-43 dye by endocytosis. After this, the NRNs were washed gently with $37^{\circ} \mathrm{C} 0.9 \% \mathrm{NaCl}$ to remove the extracellular FM1-43 dye. Finally, $70 \mathrm{mM} \mathrm{KCl}$ was added again to elicit synaptic vesicle exocytosis. The FM1-43 fluorescent dye signal was monitored by confocal microscopy (Olympus FV1000, Japan), and fluorescence images were acquired every $1 \mathrm{~s}[32,33]$.

\section{Primary hippocampal and cortical neuron culture}

Rat pups from postnatal days 1-3 $\left(\mathrm{P}_{1-3}\right)$ were first anaesthetized with isoflurane and decapitated, and then the hippocampi and cortices were removed and placed in cold phosphate-buffer solution (PBS, Solarbio, Cat. No. P1010, Beijing). After dissection and trituration, tissues were digested in $0.25 \%$ trypsin digestive solution (Beyotime, Cat. No. C0201, Shanghai, China) for $15 \mathrm{~min}$. Single cells were removed after terminating digestion using culture media containing DMEM (HyClone, Cat. No. SH30022.01, Logan, UT, USA) with 10\% fetal bovine serum (FBS, HyClone, SH30070.02, Logan, UT, USA) and $1 \%$ penicillin-streptomycin (Beyotime,Cat. No. 
C0222, Shanghai, China). The cells were then plated on cell plates precoated with $10 \mu \mathrm{g} / \mathrm{mL}$ poly-D-lysine (PDL, No. P0899 Sigma, Saint Louis, CA, USA) and cultured in culture media containing neurobasal medium (Invitrogen, Cat. No. 21103049, Carlsbad,USA) with 2\% B27 supplement (Invitrogen, Cat. No. 17504004, Carlsbad, CA, USA) without FBS. After $3 \mathrm{~d}$, the neurons were treated with $5 \mu \mathrm{M}$ cytosine arabinoside (Sigma, Cat. No. C8768, USA) to inhibit astrocyte proliferation. For the western blot and qRT-PCR experiments, the neurons were used 5-7 d after plating. For the FM1-43 dye experiments, the neurons were plated on cover glass precoated with $10 \mu \mathrm{g} / \mathrm{mL}$ PDL and cultured for $12 \mathrm{~d}$. Half the volume of culture media was changed every three days [3].

\section{Dual luciferase reporter assay}

Plasmid construction was performed according to the method previously described [3, 34]. Genome DNA templates were first extracted from rat tails with Wizard ${ }^{\circ}$ genome DNA purification kit (Promega, Cat. \#A1125), and DNA electrophoresis was used to verify the quality of extracted genomic DNA using DL2000DNA marker (Fig. S1a). The sequences of the full-length 3' untranslated region (3'UTR) and mutant 3'UTR of the Snap25, Vamp2, Stx1a and, Syt1 genes 3 are shown in Additional file 1. Amplification of the acquired genomic DNA was performed by PCR and the PCR products were recollected with a DNA gel extraction kit (Dongsheng Biotech) (Fig. S1a-e). The primers for the amplification of the full-length 3'UTR of the Snap25, Vamp2, Stx1a, and Syt1 genes are shown in Table S1. Next, the purified PCR products were incubated with psiCHECK-2 vectors using the $X h o I$ and NotI enzyme for enzymatic digestion. The enzyme digestive products were purified on an agarose gel with a DNA Gel extraction kit and ligated into psiCHECK-2 vectors using T4 DNA ligase (TaKaRa, D2011A). The ligated products were transformed into E.coli DH5 $\alpha$ competent cells. The positive recombinant clones were screened by agarose gel electrophoresis and BLAST analysis of cloned DNA sequences relative to the 3'UTRs of the genes published in NCBI was employed to verify vector construction (Fig. S2).

Mutagenesis of nucleotides was carried out using direct oligomer synthesis for the 3'UTR region of Syt1, Stx1a, Snap25 and Vamp2. Point mutations were introduced into a possible miR-153 binding site located in the 3'UTR region. Plasmid construction for carrying the mutated 3'UTRs of these four genes were constructed using the same method as that used for wild-type plasmid construction. The PCR primers for the mutagenesis of the 3'UTRs of these four genes are shown in Table S1.

HEK293T cells $\left(2 \times 10^{4}\right.$ cells $)$ were inoculated on 24 well plates at $50 \% \sim 60 \%$ confluences. These cells were then transfected with $20 \mu \mathrm{mol} / \mathrm{L}$ miR-153 mimics, AMO- 153, or mis- miR-153 siRNAs as well as $0.5 \mu \mathrm{g}$ psi-CHECK ${ }^{\mathrm{Tm}}$-2-target DNA (Renilla luciferase vector) and $1 \mu \mathrm{L}$ blank plasmid using Lipofectamine 2000 (Invitrogen, USA) transfection reagent according to the manufacturer's instructions. After $48 \mathrm{~h}$ of transfection, luciferase activities were measured with a dual luciferase reporter assay kit (Cat. No. E1910, Promega, USA) and luminometer $\left(\right.$ GloMax $^{\mathrm{ma}}$ 20/20, Promega, USA); Nucleotide-substitution mutagenesis was carried out using direct oligomer synthesis for the 3' UTRs of Stxla, Snap25, Vamp2 and Syt-1. All constructs were sequence verified.

\section{Transfection procedures}

After six days of cell culture, $75 \mathrm{pmoL} / \mathrm{mL}$ miR-153 mimics and/or AMO-153, oligodeoxynucleotides (ODNs), scrambled miR-153 or scrambled AMO-153 (Table S2) were transfected into cultured neonatal hippocampal and cortical neurons with X-treme GENE siRNA transfection reagent (catalog\#04476093001, Roche, USA) according to the manufacturer's instructions. Forty-eight hours after transfection, cells were collected for subsequent total RNA isolation, protein purification or FM1-43 staining. Transfection was performed at $12 \mathrm{~d}$ for the FM1-43 dye experiments [3].

\section{Immunofluorescence detection}

After $48 \mathrm{~h}$, the cultured cells were fixed in $4 \%$ paraformaldehyde for $30 \mathrm{~min}$. After the cells were blocked, they were incubated with the Tuj1 antibody (Cat.\#T8578; 1 : 200; Sigma, USA) to label the neurons and then the cultured cells on the glass were washed and incubated with the secondary antibodies conjugated to Alexa Fluor 488 (Invitrogen) for $1 \mathrm{~h}$ at $37^{\circ} \mathrm{C}$. After incubation with secondary antibody and DAPI as usual, neurons were mounted on coverslips to obtain confocal images by FluoView $^{\text {Tm }}$ FV300 (Olympus) using $\times 60$ objective with the same condition at a resolution of $1024 \times 1024$ pixels (12 bit) [27].

\section{qRT-PCR}

Total RNA was purified with the Trizol reagent (Invitrogen, Carlsbad, CA, USA) according to the manufacturer's instructions. The extracted RNA was then reverse transcribed into cDNA according to the introduction of the ReverTra Ace ${ }^{\circ}$ qPCR RT Kit (Code: FSQ-101, Osaka, Japan). The miR-153 level was assessed using the FastStart Universal $\mathrm{SYBR}^{\circ}$ Green Master kit (Roche Diagnostics $\mathrm{GmbH}$, Mannheim, Germany). U6 was used as an internal control of miRNAs and actin was selected as the internal reference mRNA. The qRT-PCR probes and primers for miR-153, miR-137, miR-34c, miR-135a, and $U 6$ were designed by Invitrogen (Table S3). QRT- 
PCR was performed on a LightCycle 96 Instrument (Roche Diagnostics $\mathrm{GmbH}$, Mannheim, Germany), and the protocol was as follows: 1.preincubation: $95^{\circ} \mathrm{C}, 10$ min; 2.amplification: (1) denaturation: $95^{\circ} \mathrm{C}, 15 \mathrm{~s}$;(2) anneal: $\left.60^{\circ} \mathrm{C}, 30 \mathrm{~s} ; 3\right)$ extension: $72{ }^{\circ} \mathrm{C}, 30 \mathrm{~s} ; 3$. cooling: $37^{\circ} \mathrm{C}, 30 \mathrm{~s}$. Repeat "2" for 40 cycles. The results were normalized against the internal control using the $\delta-\delta$ CT method [3].

\section{Western blot}

The concentrations of the protein extracts were measured using the Bio-Rad Protein Assay kit (Bio-Rad, Hercules, CA, USA) with bovine serum albumin standards. Protein samples fractionated on a $10 \%$ sodium dodecyl sulfate polyacrylamide gel electrophoresis gel were transferred to a nitrocellulose membrane. Table S4 displays all of the primary antibodies used in this study. Western blot bands were captured using the Odyssey Infrared Imaging System (LI-COR Biosciences, Lincoln, NE, USA) and quantified using Odyssey version 3.0 software by measuring the band intensity (area - optical density (OD)). Protein expression in each group was normalized to the internal control $\beta$-actin (1:1000, G8795, Sigma).

\section{Human blood sample preparation}

Vascular dementia patients (between $70 \sim 90$ years old), from the Second Affiliated Hospital of Harbin Medical University, were recruited for this experiment based on the criteria of the National Institute of Neurological and Communicative Disorders (NINCDS) (Sarazin, etal., 2012). Written consent was obtained from all subjects, and the study protocol was approved by the Ethics Committee of Harbin Medical University (HMUIRB20140029). To prepare plasma samples for miRNA evaluation, whole blood samples ( $2.5 \mathrm{~mL}$ per patient) were collected from subjects via a direct venous puncture into tubes containing sodium citrate and centrifuged at $1000 \times \mathrm{g}$ for $5 \mathrm{~min}$, and then the supernatant (plasma) was carefully transferred into an RNase-free tube for RNA extraction [35].

\section{Statistical analysis}

All data are represented by the mean and standard error of the mean (s.e.m). Each data set was analysed for its ability to meet the statistical assumptions for equality of the variance. The independent sample test was calculated using the Levene variance equality test. If $P>0.05$, independent Student's t-test was used for the comparison between two groups; if $P<0.05$, the Kruskal-Wallis rank sum test was performed. One-way ANOVA was performed for the comparison among multivariate groups, and post hoc analyses of significant main effects were further examined using Fisher's procedures for learning systems design (PLSD) tests. For repeated measures data, Mauchly's test of sphericity was first performed to evaluate the relationship among the repeatedly measured data. If $P>0.05$, a general linear model was selected for further analysis; if $P<0.05$, GreenhouseGeisser corrected results or multivariate ANOVA was used for further analysis of multiple comparisons. All statistical analyses were performed in SPSS software (version 11).

\section{Results}

\section{$\mathrm{CBH}$ impairs presynaptic function in rats}

To evaluate presynaptic plasticity in the hippocampi of $\mathrm{CBH}$ rats, we performed $2 \mathrm{VO}$ surgery on rats $[3,36]$. As shown in Fig. 1a and b, similar to our previous study [3], 2VO surgery elicited markedly low cerebral blood flow $(\mathrm{CBF})$ at 8 weeks $(8 \mathrm{~W})$, as indicated by both TTC staining and fMRI evaluation. We then prepared brain slices and monitored the fEPSP in the stratum radiatum of CA1 by electrically stimulating the $\mathrm{SC}$ of the CA3 pathway (Fig. 1c). Based on the input-output (I-O) curve, compared with the sham group, the $2 \mathrm{VO}$ group exhibited reduced fEPSP responses (Fig. $1 \mathrm{~d}, P=0.002$ ). For example, the fEPSP amplitude in the $2 \mathrm{VO}$ rat slices was $\sim 75 \%$ of that observed in the sham controls when the stimulus intensity was $60 \mu \mathrm{A}$, and the percentage was further decreased to $\sim 60 \%$ at $120 \mu \mathrm{A}$ stimulation (Fig. 1e, $P=$ 0.003). To evaluate the presynaptic vesicle release probability, PPF $(60 \mu \mathrm{A})$ measurements were performed and evaluated by PPR [30]. The PPR in the brain slices of the 2VO group, compared with that of the sham controls, was significantly increased when the interstimulus interval (ISI) ranged from $20 \sim 100 \mathrm{~ms}$ (Fig. 1f, $P<0.0001$ ). The PPR was $1.48 \pm 0.01$ in the sham group and $1.85 \pm 0.02$ in the $2 \mathrm{VO}$ rats when the ISI was $40 \mathrm{~ms}$ (Fig. $1 \mathrm{~g}, P<0.0001$ ). These findings demonstrate an impaired presynaptic function in the $2 \mathrm{VO}$ rats.

Previous elegant studies have demonstrated that synaptic vesicle fusion is a crucial step for presynaptic neurotransmitter release triggered by the influx of $\mathrm{Ca}^{2+}$, which is controlled by the core fusion machinery composed of the SNARE-complex and Sec1/Munc18-like (SM) proteins [12, 37] (Fig. 1h). We speculated that synaptic vesicle fusion-related proteins might be changed under $\mathrm{CBH}$ conditions. Western blotting experiments showed that the protein levels of all three SNAREcomplex proteins (SNAP-25, $P=0.0017$; syntaxin-1A, $P=0.005$ and VAMP-2, $P=0.02)$ and the $\mathrm{Ca}^{2+}$-sensor protein Syt1 $(P=0.015)$ were significantly lower in the hippocampi of $2 \mathrm{VO}$ rats than in those of sham rats (Fig. $1 \mathrm{i}$ and $\mathrm{j}$ ). However, the protein levels of Munc-13, Munc-18-1 and Complexin1/2, coregulators of vesicle exocytosis, were unchanged (Fig. $1 \mathrm{k}$ and $1, P>0.05$ ). These findings suggest that the decreased protein expression of SNARE complex proteins and Syt1 may 


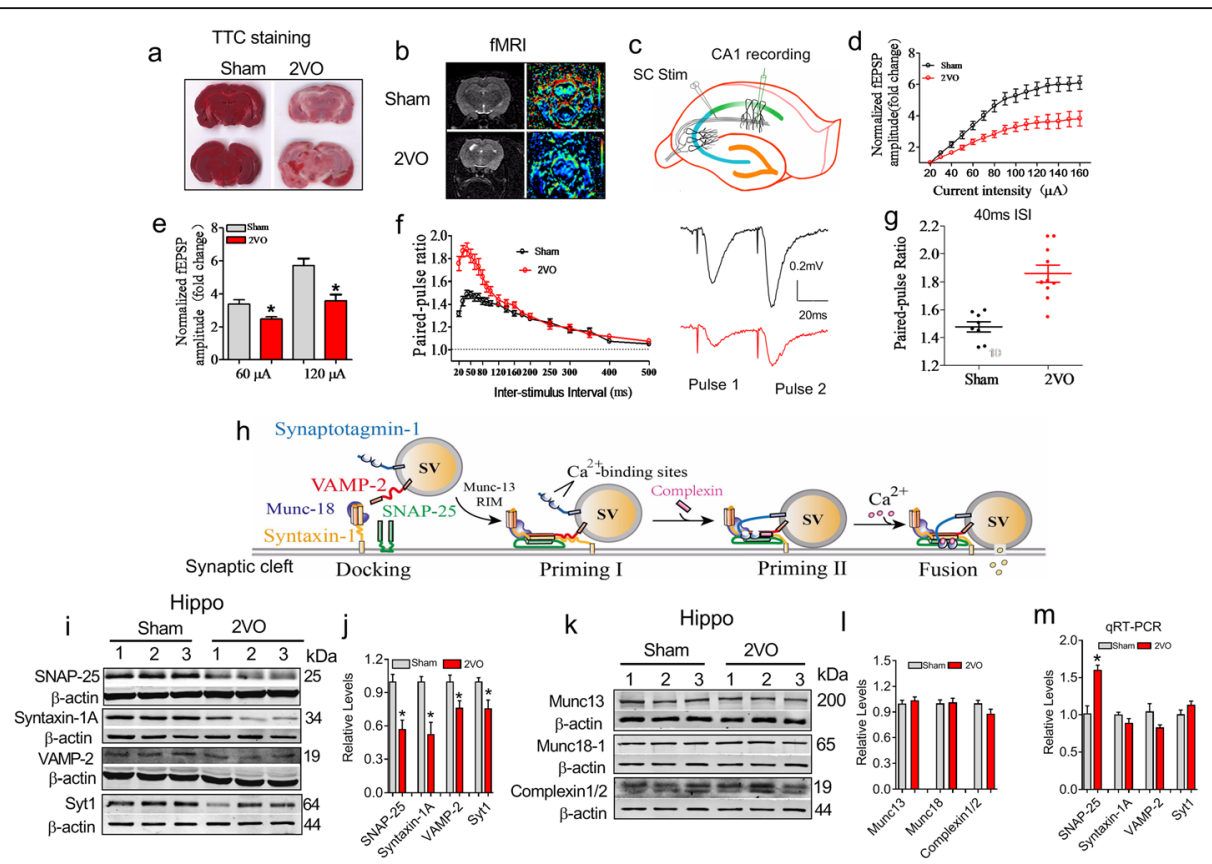

Fig. 1 Impairment of presynaptic vesicle release in CBH rats $8 \mathrm{~W}$ following $2 \mathrm{VO}$ surgery. a $T \mathrm{CC}$ staining was used to identify brain ischaemia in rats at $8 \mathrm{~W}$ after $2 \mathrm{VO}$. Red represents normal tissue and white represents infarct tissue. $\mathbf{b}$ fMRI brain images of rats at $8 \mathrm{~W}$ after $2 \mathrm{VO}$. fMRI, functional magnetic resonance imaging. c Schematic diagram showing the placement of the recording (CA1) and stimulating electrodes (CA3) in hippocampal slices. $\mathbf{d}$ Comparison of $\mathrm{I}-\mathrm{O}$ curves between sham and $2 \mathrm{VO}$ rats. $\mathrm{F}(14,196)=15.011, P<0.002 . n=8$ rats. e Fold-change differences in the fEPSP amplitude between the 2VO and sham groups. $P_{\text {Levene }}=0.713$, Student's t-test: $P=0.003 . n=8$. $\mathbf{f}$ Scatter plot summarizing the PPR in sham and $2 \mathrm{VO}$ rats. Right Insertion: sample of fEPSP traces in the sham and $2 \mathrm{VO}$ rats with a $40 \mathrm{~ms}$ ISI stimulated by $60 \mu \mathrm{A}$. $X^{2}$ Mauchly $=520.339, \mathrm{~F}$ $(18,379)=19.122, P<0.0001 . n=8$ slices for sham rats, $n=10$ for 2 VO rats. $\mathbf{g}$ Scatter dot plot showing the increased PPR in the 2 VO rats compared with that in sham rats with a $40 \mathrm{~ms}$ ISI. Each dot represents one data point from one slice. $P_{\text {Levene }}=0.073$, Student's t-test: $P<0.0001$. $\mathbf{h}$ Diagram depicting a molecular model of the synaptic vesicle exocytosis process. $\mathbf{i} \mathbf{j}$ Expression of SNAP-25, syntaxin-1A, VAMP-2 and Syt1 proteins in the hippocampal synaptosomes of 2 VO rats. $n=6$ rats. SNAP-25: $P_{\text {Levene }}=0.719$, Student's t-test, $P=0.0017$; syntaxin- 1 A: $P_{\text {Levene }}=0.002$. Kruskal-Wallis rank sum test: $P=0.005 ;$ VAMP-2: $P_{\text {Levene }}=0.843$, Student's t-test, $P=0.02 ;$ Syt1: $P_{\text {Levene }}=0.477$, Student's t-test, $P=0.015$. ${ }^{*} P<0.05$. $\mathbf{k}-\mathbf{I}$ Protein expression of Munc-13, Munc-18-1 and complexin 1/2 in hippocampal synaptosomes of 2VO rats. Student's t-test, $P>0.05$. m Relative mRNA levels of SNAP-25, syntaxin-1A, VAMP-2 and Syt- 1 in the hippocampi of 2 VO rats (Student's t- test: SNAP-25, $P=0.025 ;$ syntaxin-1A, $P=0.303$; VAMP-2, $P=0.361 ;$ Syt-1, $P=0.287) . \mathrm{n}=6$ rats. ${ }^{*} P<0.05$ vs sham

participate in the impaired presynaptic vesicle release observed in $\mathrm{CBH}$ rats.

\section{MiR-153 targets SNARE complex-related genes and the Syt1 gene}

Compared to the levels in sham rats, the SNARE complex protein and Syt1 protein levels were decreased in $2 \mathrm{VO}$ rats, while their mRNA levels were not reduced, indeed, and the level of SNAP25 mRNA was even increased in $2 \mathrm{VO}$ rats (Fig. $1 \mathrm{~m}, P=0.025$ ). Therefore, we considered that a posttranscriptional regulatory mechanism might be involved in the observed changes. Multiple miRNAs have been previously reported to regulate synaptic fusion-related proteins at the mRNA level. For example, miR-153 regulates Snap25, Vamp2, Snca, Trak2, Bsn and Pclo genes at the mRNA level [23]; miR-137 inhibits complexin-1, Nsf and Syt1 in mouse model of schizophrenia [20]; miR-34c targets VAMP-2 [21] and miR-135 $a$ binds the complexin-1 and complexin- 2 genes in the amygdala [22]. To identify which one of the above
miRNAs is involved in the $\mathrm{CBH}$ induced downregulation of fusion-related proteins in the hippocampi of rats, we performed qRT-PCR test. As displayed in Fig. 2a, compared to the sham rats, $2 \mathrm{VO}$ rats exhibited $\sim 2$-fold increase in the expression of $m i R-153(P=0.011)$, however, the $m i R-137$ and $m i R-135 a$ levels were unchanged $(P>0.05)$. In contrast, $m i R-34 c$ expression was reduced in the hippocampi of $2 \mathrm{VO}$ rats. These data suggest that the decreased expression of SNAP-25 or VAMP2 in the hippocampi of $2 \mathrm{VO}$ rats may result from upregulated expression of $m i R-153$, but do not correlate with the observed downregulation of $m i R-34 c$. Additionally, the decrease in Syt1 protein expression was not associated with miR-137 expression. Furthermore, to elucidate the potential clinical significance of $m i R-153$, 12 male subjects (7 controls and $5 \mathrm{VaD}$ patients) with higher education backgrounds were recruited to our study and assessed by the Mini-Mental State Examination (MMSE), Montreal Cognitive Assessment (MoCA) and Hamilton Depression Scale (HAMD) (Table S5). As 


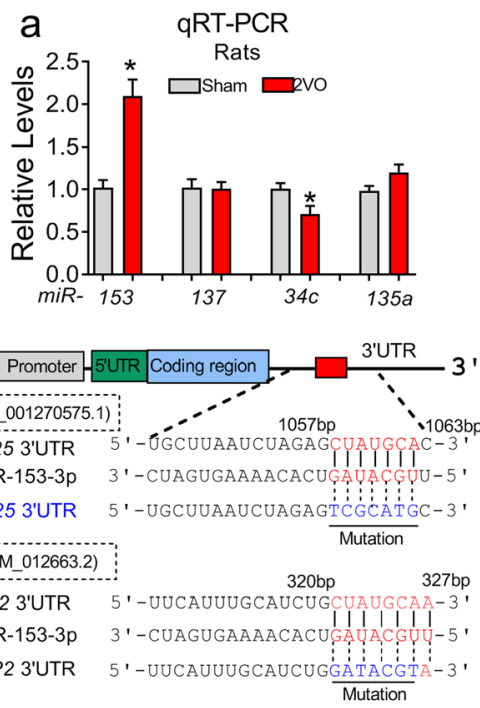

d Luciferase Assay-Snap25

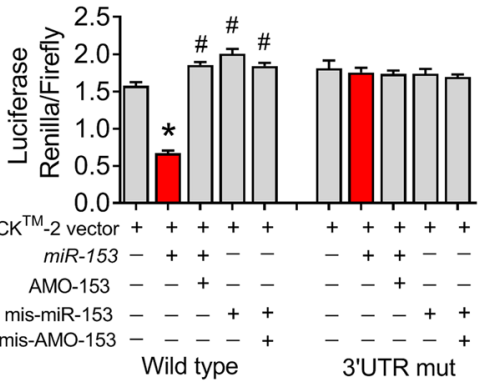

f

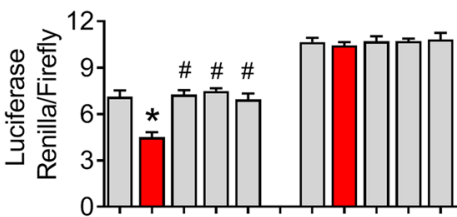

psiCHECK $^{\mathrm{TM}}-2$ vector ++++++++++ miR-153 $-++-\quad++--$

AMO-153 - -+---++-

$\operatorname{mis-miR-153}--+++---++$
mis-AMO-153 - - - + ---+

Wild type 3'UTR mut
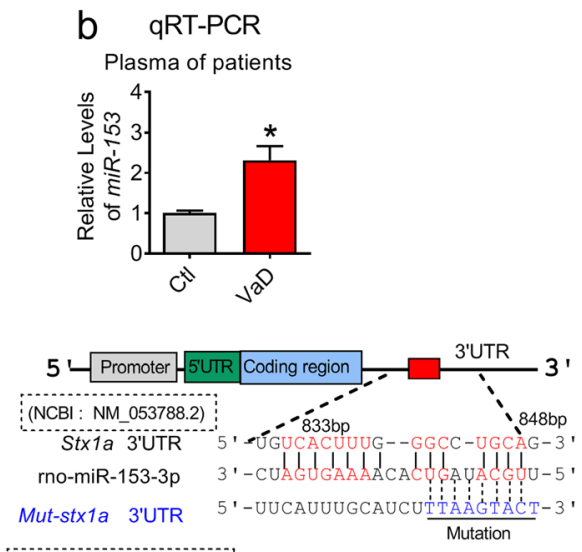

(NCBI - NM $001033680 .-$.

Syt 13 'UTR 5'-UCAGAACUGA 1622bp 1628bp

no-miR-153-3p 5'-UCAGAACUGACAGTI I I I - ${ }^{\prime}$

Mut-Syt 1 3'UTR 3'-CUAGUGAAAACACUGAUACGUU'-5'

Mutation

e

Luciferase Assay-Vamp2

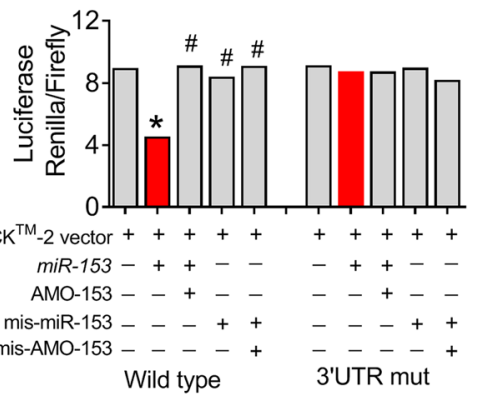

g Luciferase Assay-Syt1

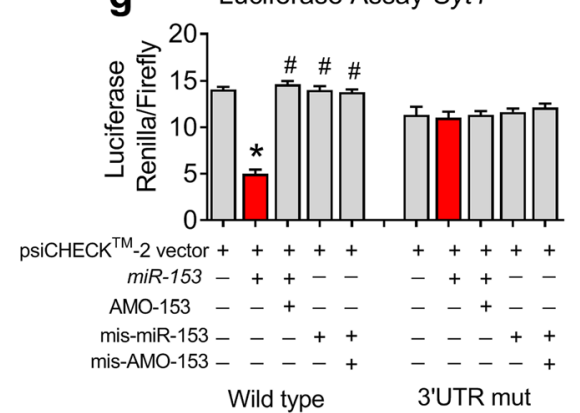

Fig. 2 MiR-153 targets the 3'UTRs of the Snap25, Vamp2, Stx1 $a$ and Syt1 genes. a The levels of miR-153, miR-137, miR-34C and miR-135a in the hippocampi of sham and 2VO rats as assessed by qRT-PCR (for miR-153: $P_{\text {Levene }}=0.008$, Student's t-test: $P=0.011$ ), $n=6$. b MiR-153 levels in the plasma of VaD patients as assessed by qRT-PCR $\left(P_{\text {Levene }}=0.002\right.$, Student's t-test, $\left.P=0.019\right)$. VaD: vascular dementia. c Complementarity between the miR-153 seed sequence and the 3'UTRs of Snap25, Vamp2, Stx1a and Syt1 predicted using the TargetScan5.1 algorithm and RNAhybrid database. The gene mutations are underlined. $3^{\prime} U T R, 3^{\prime}$ untranslated region. $\mathbf{d - g}$ Dual luciferase assay of interactions between miR-153 and predicted binding sites or mutated binding sites in the 3'UTR of Snap25 (wild-type: $P_{\text {Levene }}=0.192$, one-way ANOVA: $F=85.093, P<0.0001 ; 3^{\prime} U T R$ mutant ( $3^{\prime}$ UTR mut): $P_{\text {Levene }}=0.184$, one-way ANOVA: $F=0.199, P=0.933$ ), Vamp2 (wild-type: $P_{\text {Levene }}=0.168$, one-way ANOVA: $F=55.704, P<0.0001$; 3'UTR mut: $P_{\text {Levene }}=0.041$, one-way ANOVA: $F=2.561, P=0.104$ ), Stx 1 a (wild-type: $P_{\text {Levene }}=0.276$, one-way ANOVA: $F=119.138, P<0.0001 ; 3^{\prime} U T R$ mut: $P_{\text {Levene }}=0.0331$, one-way ANOVA: $F=0.155, P=0.957$ ) and Syt1 (Wild-type: $P_{\text {Levene }}=0.522$, one-way ANOVA: $F=825.5700, P<0.0001 ; 3^{\prime} U$ TR mut: $P_{\text {Levene }}=0.077$, one-way ANOVA: $F=1.603, P=0.248$ ) in HEK293T cells. $n=3$ batches per group. Fisher's PLSD test was used for the post hoc analyses of the two-group comparisons. ${ }^{*} P<0.05$ vs control or psiCHECK ${ }^{\mathrm{TM}}-2$ vector; ${ }^{\#} P<0.05$ vs miR-153 
shown in Fig. 2b, the levels of miR-153 in the plasma of $\mathrm{VaD}$ patients were significantly higher than those in the plasma from the control group $(P=0.019)$, indicating that miR-153 is potentially involved in the pathophysiological process of human presynaptic vesicle release disorders. Bioinformatics methods (TargetScan Human 5.1 and RNAhybrid database) were then employed to identify the potential miRNAs regulating Syt1 and syntaxin1A expression. Surprisingly, we found that $m i R-153$ alone had conserved binding sites in the 3'UTRs of the Snap25 and Vamp2 genes as well as targets in the 3'UTRs of the Stx1a and Syt1 genes (Fig. 2c).

To better understand how miR-153 regulates all four genes, we first performed a dual luciferase reporter gene assay to evaluate the binding ability of $m i R-153$ with these genes. The full-length 3'UTRs of these four genes containing the miR-153 binding sites were separately cloned, purified, and ligated into the psiCHECK ${ }^{\mathrm{m}}-2$ vector at the site between the Renilla luciferase gene and the synthetic poly (A) tail (Fig. S1a-e and S2a-c). Thereafter, the effects of miR-153 on reporter activities were assessed in HEK293T cells. As predicted, cotransfection of chemically synthesized miR-153 mimics and the
psiCHECK $^{\mathrm{m}-2}$ vector plasmid consistently produced lower Renilla luciferase activities than transfection of the empty psiCHECK ${ }^{\mathrm{tm}}-2$ vector plasmid alone (Fig. $2 \mathrm{~d}-\mathrm{g}, P$ $<0.0001$ ). The silencing effect of $m i R-153$ reached $\sim 60 \%$ on the Snap25 transcript (Fig. 2d), $~ 50 \%$ on the Vamp2 transcript (Fig. 2e), 35\% on the Stx1a transcript (Fig. 3f) and $\sim 70 \%$ on the Syt1 transcript (Fig. $2 \mathrm{~g}$ ), and these effects could be prevented by cotransfection of the psiCHECK $^{\mathrm{m}}-2$ vector plasmids with AMO-153. However, the transfection of psiCHECK ${ }^{\mathrm{m}}-2$ vector plasmids with scrambled oligoribonucleotides of either mis-miR-153 or antisense mis-AMO-153 in all four genes failed to affect luciferase activity (Fig. 2d-g). Furthermore, when we cloned the full-length 3'UTR of the Snap25 (1057-1063 bp), Vamp2 (320-327 bp), Stx 1a (841-848 bp) and Syt1 (1622-1628 bp) with mutant binding sites (Fig. 2c), the repressive effects of $m i R-153$ on the luciferase activities of these genes were abolished (Fig. $2 \mathrm{~d}-\mathrm{g}, P>0.05$ ). These results suggest that miR-153 directly target the 3'UTRs of the Snap25, Vamp2, Stx1a and Syt1 genes.

To confirm that miR-153 gain-of-function affects the expression of these four proteins in neurons, chemically synthesized miR-153 mimics and AMO-153 were

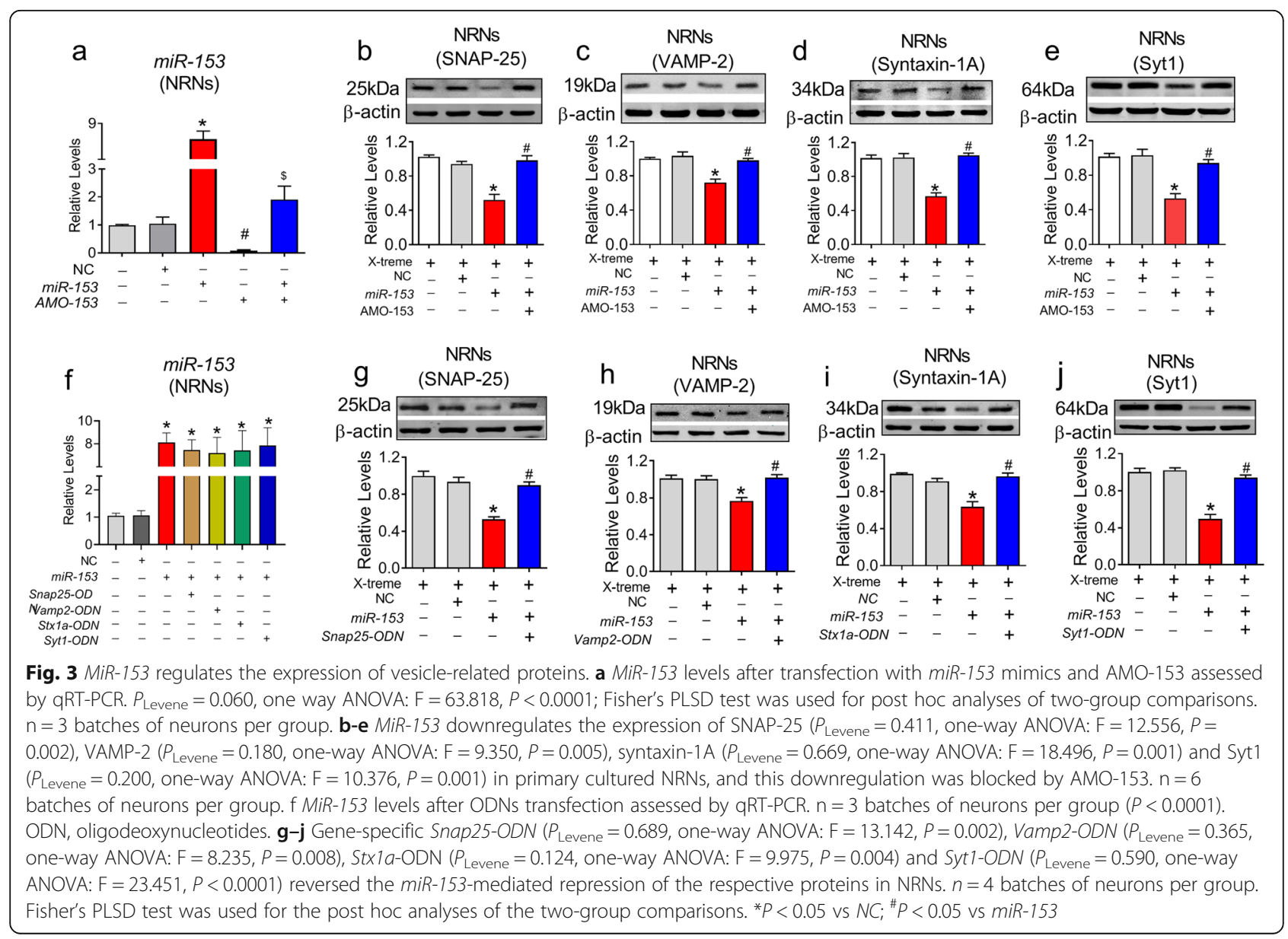


successfully transfected into cultured NRNs by the Xtreme GENE siRNA transfection reagent (Fig. 3a, $P<$ 0.0001 and Fig. S3). The miR-153 mimics effectively inhibited the expression of all four proteins with an inhibitory efficiency of $\sim 48 \%$ for SNAP-25 $(P=0.002)$, $47 \%$ for Syt1 $(P=0.001), \sim 43 \%$ for syntaxin-1A $(P=$ $0.002)$ and $\sim 28 \%$ for VAMP-2 $(P=0.005)$, whereas the scrambled NC failed to affect the levels of these proteins (Fig. 3b - e). In contrast, AMO-153 rescued the downregulation of all proteins elicited by the miR-153 mimics. Subsequently, to confirm that miR-153 mediates the downregulation effect on these four proteins by directly targeting the binding sites of the four genes, the miRNA-masking antisense ODNs (miRmasking) technique was employed as previously reported [3]. An ODN is an antisense oligodeoxynucleotide fragment designed to fully base pair to a protein-coding mRNA at the sequence motif spanning the binding site for an endogenous miRNA of interest. Since a miR-mask only acts on the target gene with minimal effects on other target genes that may also be targeted by a same miRNA, the anti-miRNA action of a miR-mask is gene-specific. Based on this mechanism, we designed four miR-153 masks that could base pair the miR-153 binding sites in the 3'UTRs of the Snap25, Vamp2, Stx1a and Syt1 genes and labelled these masks Snap25-ODN, Vamp2-ODN, Stx1a-ODN and Syt1-ODN, respectively, and evaluated whether these ODNs could shield the action of $m i R$ 153 on the four genes. As predicted, after successful transfection of the four ODNs (Fig. 3f), these ODNs effectively blocked the repressive effects of $m i R-153$ on the protein expression of SNAP-25 $(P=0.002)$, syntaxin-1A $(P=0.004)$, VAMP-2 $(P=0.008)$ and Syt1 $(P<0.0001)$ (Fig. 3g-j), highlighting the sequence specificity of the actions of $m i R-153$.
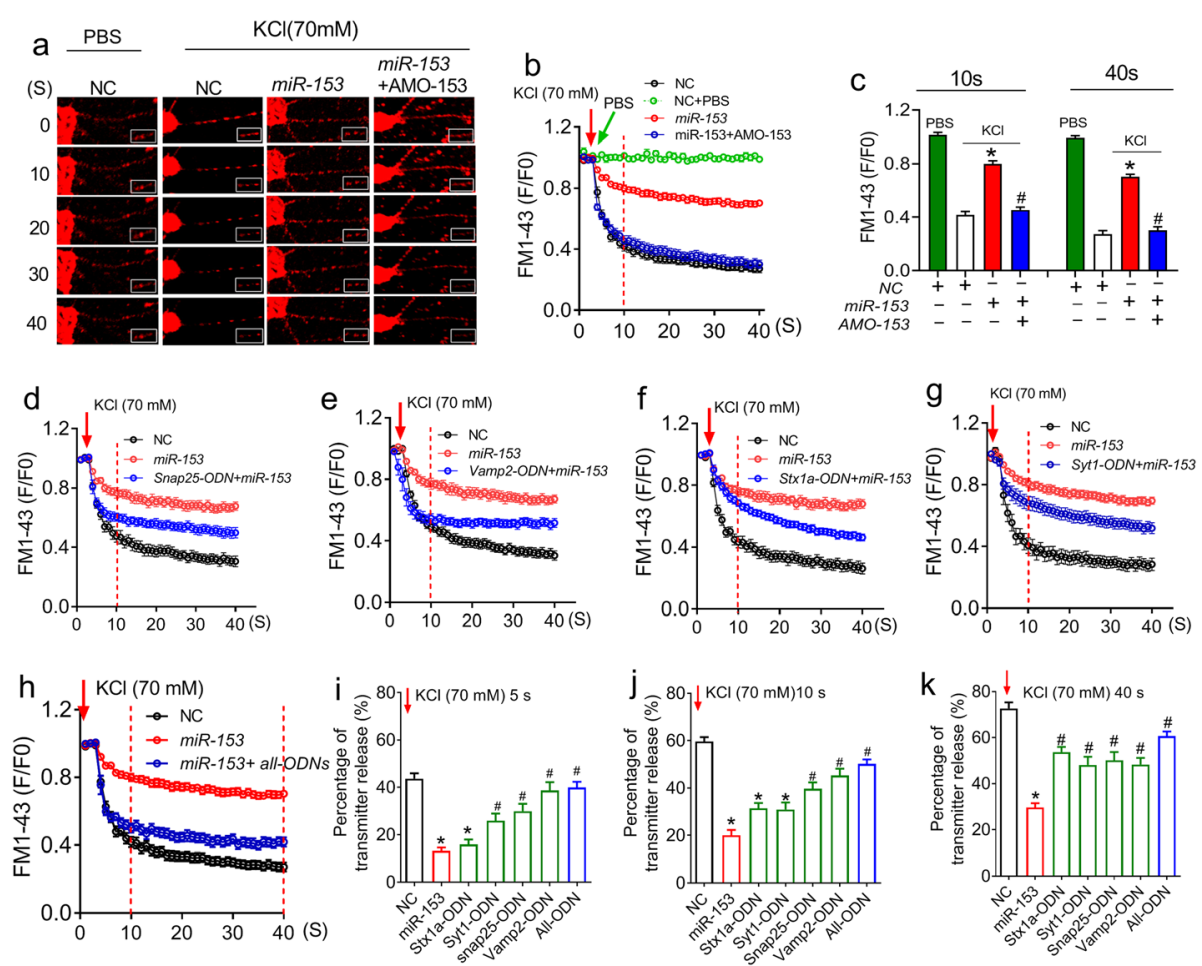

Fig. 4 MiR-153 represses synaptic vesicle exocytosis by targeting vesicle release-related proteins. a FM1-43 fluorescent dye images showing presynaptic boutons in neurons before stimulation ( $\mathrm{t}=0 \mathrm{~s}$ ) and following PBS or $70 \mathrm{mM} \mathrm{KCl}$ stimulation at 10, 20, 30 , and $40 \mathrm{~s}$. The insert shows enlarged synaptic boutons from the framed area in the main panels. b FM1-43 fluorescent dye imaging analysis $\left(X^{2}\right.$ Mauchly $=3068.620, P<0.0001$; $\left.F_{\text {total }(39,2146)}=69.973, P<0.0001\right)$. $\mathbf{c}$ Bar graph of FM1-43 staining normalized fluorescence density at $10 \mathrm{~s}\left(P_{\text {Levene }}=0.330\right.$, one-Way ANOVA: $F=$ 46.738, $P<0.0001)$ and $40 \mathrm{~s}$ ( $P_{\text {Levene }}=0.105$, one-way ANOVA: $\left.F=90.788, P<0.0001\right)$ following $70 \mathrm{mM} \mathrm{KCl}$ stimulation. $n=17$ synaptic boutons from 3 batches of NRNs in each group. $\mathbf{d}$-h FM1-43 fluorescence intensity curve showing the action of Snap25-ODN $\left(\mathbf{d}, F_{\text {total }}(39,1132)=27.987\right.$, $P<0.0001)$, Vamp2-ODN (e, $\left.F_{\text {total }}(39,1132)=32.628, P<0.0001\right)$, Stx1a-ODN $\left(\mathbf{f}, F_{\text {total }}(39,1132)=40.414, P<0.0001\right)$, Syt1-ODN $\left(\mathbf{g}, F_{\text {total }}(39,1132)=\right.$ $32.183, P<0.0001)$ or the four ODNs combined $\left(\mathbf{h}, F_{\text {total }}(39.2029)=25.076, P<0.0001\right)$ on FM1-43 fluorescent dye exocytosis. $\mathbf{i}-\mathrm{k}$ Statistical analysis of the effects of the SNAP-25, VAMP-2, syntaxin-1A and Syt1 proteins on vesicle release. Bar graph showing the percentage of released vesicles after $70 \mathrm{mM} \mathrm{KCl}$ stimulation at $5 \mathrm{~s}$ (i: $P$ Levene $=0.357$, one-way ANOVA: $F=15.657, P<0.0001), 10 \mathrm{~s}\left(\mathrm{j}: P_{\text {Levene }}=0.027\right.$, one-way ANOVA: $\mathrm{F}=24.181$, $P<0.0001)$ and $40 \mathrm{~s}\left(\mathbf{k}: P_{\text {Levene }}=0.142\right.$, one-way ANOVA: $\left.F=33.132, P<0.0001\right) . n=12$ synaptic boutons from 3 batches of NRNs in each group. Fisher's PLSD test was used for the post hoc analyses of the two-group comparisons. ${ }^{*} P<0.05$ vs NC; ${ }^{\#} P<0.05$ vs miR-153 
MiR-153 regulates synaptic vesicle release by targeting SNARE complex-related genes and the Syt 1 gene in vitro To determine whether miR-153 gain-of-function impairs synaptic vesicle release, we loaded NRNs with FM1-43 fluorescent dye, which can be used to monitor vesicle release by labelling synaptic vesicles [32]. As illustrated in Fig. 4a and b, after treatment with phosphate buffer saline (PBS), the red fluorescent signal of the synaptic boutons was not changed in the NRNs transfected with NC, which was sharply depleted by adding $\mathrm{KCl}$. The F/FO ratio was reduced to $\sim 0.4$ at $10 \mathrm{~s}$ and further decreased to $\sim 0.3$ at $40 \mathrm{~s}$. However, the miR-153 mimics markedly blocked the fast-declining fluorescent signal of the boutons with the F/F0 ratio as high as 0.8 at $10 \mathrm{~s}$ and 0.7 at $40 \mathrm{~s}$ after $\mathrm{KCl}$ stimulation; this effect was fully reversed by the cotransfection with AMO-153 (Fig. 4a-c, $P<0.0001$ ). Overall, these data provide evidence that $m i R-153$ gain-offunction impairs presynaptic vesicle release.

Subsequently, four miR-153 masks: Snap25-ODN, Vamp2-ODN, Stx1a-ODN and Syt1-ODN, were employed to observe the contribution of the four ODNs to the miR-153-induced impairment of vesicle release. We found that the effect of miR-153 on vesicle release was only partially prevented when any single ODN was transfected into NRNs (Fig. 4d-g). Importantly, the effect of miR-153 on vesicle release within $5 \mathrm{~s}$ was virtually entirely dependent on the SNAP-25 and VAMP-2 proteins because Snap25-ODN or Vamp2-ODN alone almost fully reversed the miR-153 induced elevation of the F/F0 ratio (Fig. 4d, e and I, $P<0.0001$ ). However, within this time window, no effect of Stx1a-ODN (Fig. $4 \mathrm{f}$ and i) and a weak action of Syt1-ODN (Fig. $4 \mathrm{~g}$ and i, $P<0.0001$ ) on the F/FO ratio were observed. Notably, similar to the performance of all four ODNs together, Vamp2-ODN alone completely reversed the blunting effect of miR-153 on vesicle release at $5 \mathrm{~s}$ (Fig. $4 \mathrm{e}, \mathrm{h}$ and i, $P<0.0001$ ). Interestingly, as the duration after $\mathrm{KCl}$ supplementation increased to $40 \mathrm{~s}$, the preservative effect of Stx $1 \mathrm{a}-O D N$ and Syt1-ODN on the miR-153-induced impairment of vesicle release was increased and became similar to the effects of Snap25-ODN and Vamp2-ODN (Fig. 4j and k). These results suggest that the miR-153 binding sites on the 3'UTRs

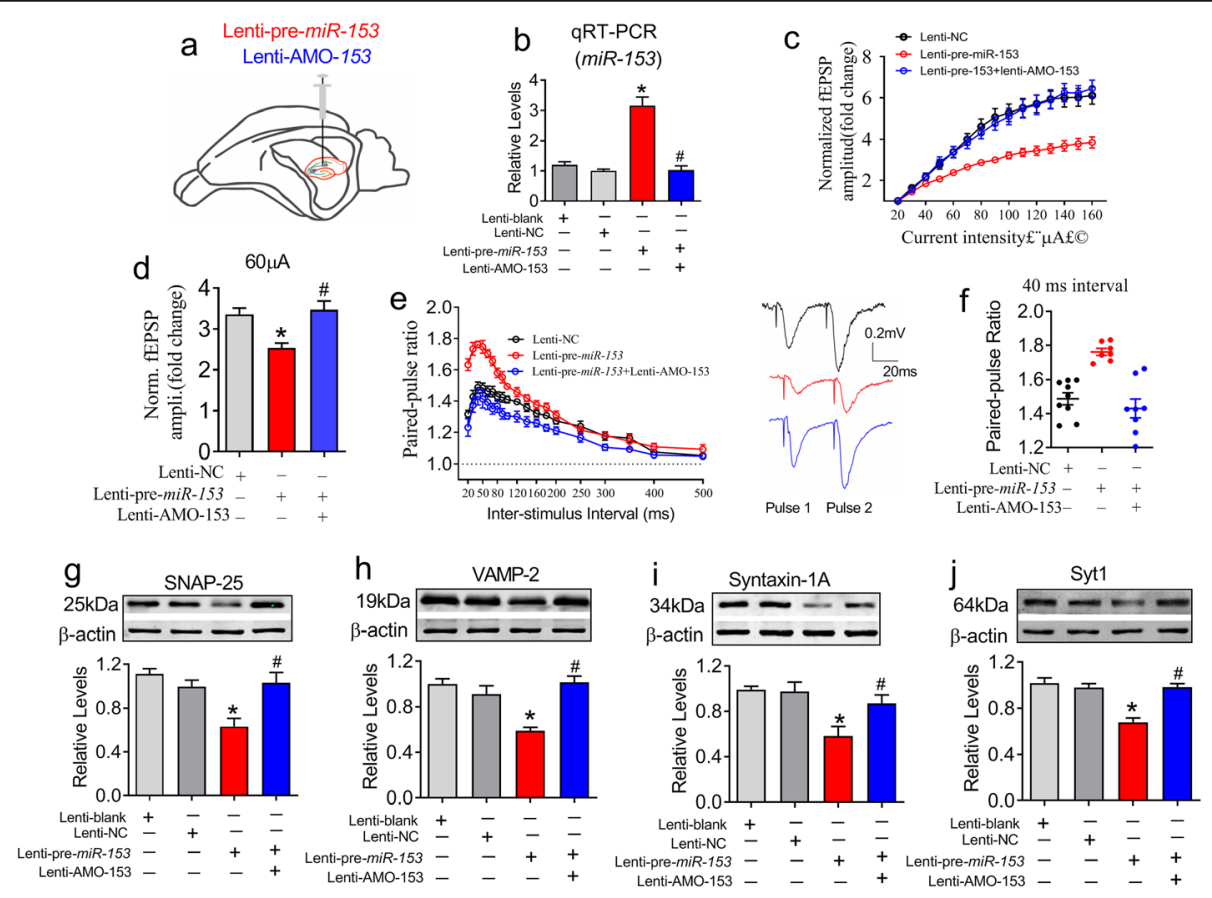

Fig. 5 MiR-153 gain-of-function impairs presynaptic vesicle release in rat hippocampi. a Schematic diagram of stereotactic lentivirus injection into the CA1 region of the hippocampus. $\mathbf{b}$ Relative levels of miR-153 in the hippocampi of rats ( $P_{\text {Levene }}=0.069$, one-way ANOVA: $F=39.14, P<0.0001$ ). $n=$ 6. $\mathbf{c}$ Lenti-AMO-153 reversed the repression effect of lenti-pre-miR-153 on fEPSP in the CA3-CA1 pathway. $X^{2}$ Mauchly $=484.594, P<0.0001 ; F_{\text {total }}(14,295)=$ $12.618, P<0.0001, n=7$ for lenti-pre-miR-153 group, $n=8$ for the lenti-AMO-153 and sham groups. $\mathbf{d}$ The fold change in the fEPSP amplitude following $60 \mu \mathrm{A}$ current stimulation ( $P_{\text {Levene }}=0.620$, one-way ANOVA: $F=3.993, P=0.032$ ). e Lenti-AMO-153 reversed the increased in the PPR induced by lenti-pre-miR-153. $X^{2}$ Mauchly $=509.893, F_{(18,433)}=18.968, P<0.0001$. Insertion: representive of EPSP traces. $\mathbf{f}$ Scatter dot plot showing the increased PPR with a $40 \mathrm{~ms}$ ISI in the lenti-pre-miR-153 injected rats compared with that in the lenti-NC-injected rats, and this increase in PPR was prevented by the coinjection with lenti-AMO-153 ( $P_{\text {Levene }}=0.186$, one-way ANOVA: $\left.F=17.454, P<0.0001\right)$. $\mathbf{g}$-j Lenti-pre-miR-153 repressed the protein levels of SNAP-25 ( $P_{\text {Levene }}=0.11$, one-way ANOVA: $\left.F=9.021, P=0.001\right)$, VAMP-2 $\left(P_{\text {Levene }}=0.352\right.$, one-way ANOVA: $\left.\mathrm{F}=13.824, P<0.0001\right)$, syntaxin-1A $(P$ Levene $=$ 0.193, one-way ANOVA: $F=7.023, P=0.002)$ and Syt $1(P$ Levene $=0.871$, one-way ANOVA: $F=17.301, P<0.0001)$ in the hippocampal synaptosomes. $n=$ 6. ${ }^{*} P<0.05$ vs lenti-NC; ${ }^{\#} P<0.05$ vs lenti-pre-miR-153 
of these four genes are involved in the impairment of presynaptic vesicle release, and VAMP-2 and SNAP-25 are the rate-limiting proteins.

\section{MiR-153 gain-of-function impairs presynaptic vesicle release in vivo}

Subsequently, we investigated whether the upregulation of $m i R-153$ affect vesicle release in vivo. We designed three lentiviral constructs, named lenti-mis-pre-miR-153 as NC (lenti-NC), lenti-pre-miR-153 and lenti-AMO-153, and stereotaxically injected them directly into the bilateral hippocampal CA1 subfields of each rat [3] (Fig. 5a and b, $P<$ 0.0001). Relative to lenti-NC injection, lenti-pre-miR-153 application resulted in an overall decrease in the fEPSP responsiveness in the CA1 area and more quickly saturated electrical stimulation as indicated by the I-O curve (Fig. $5 \mathrm{c}$, $P<0.0001$ and Fig. 6d, $P=0.032$ ), which was similar to that of the $2 \mathrm{VO}$ rat (Fig. 1c). Similarly, lenti-pre-miR-153 significantly elevated the synaptic PPR when the ISI was between $20 \mathrm{~ms}$ and $100 \mathrm{~ms}$, and this increase was prevented by coinjection with lenti-AMO-153 (Fig. 5e and f, $P<0.0001$ ). Accordingly, we observed that lenti-pre-miR-153 significantly inhibited the protein expression of SNAP-25 $(P=0.001)$, VAMP-2 $(P<0.0001)$, syntaxin-1A $(P=0.002)$ and Syt1 $(P<0.0001)$ in the rat hippocampi (Fig. $5 \mathrm{~g}-\mathrm{j})$, and these effects were reversed by lenti-AMO-153 treatment. These results indicate that miR-153 gain-of-function disturbs hippocampal vesicle release by inhibiting vesicle-related proteins in vivo.

\section{Knockdown of miR-153 rescues hippocampal presynaptic vesicle release decline in $2 \mathrm{VO}$ rats}

Because 2VO elicited increased miR-153 levels in the hippocampus of $2 \mathrm{VO}$ rats (Fig. 6a), we next sought to determine whether knockdown of miR-153 could improve the decreased presynaptic vesicle release in $2 \mathrm{VO}$ rats. As predicted, compared with lenti-NC, lenti-AMO153 significantly inhibited the high expression of $m i R$ 153 in the hippocampi of $2 \mathrm{VO}$ rats (Fig. 6a, $P=0.0001$ ). Moreover, lenti-AMO-153 significantly rescued the decreased fEPSP amplitude (Fig. 6b, $P<0.0001$ and c, $P=$ 0.002 ) and impaired synaptic facilitation (Fig. $6 \mathrm{~d}$ and e, $P<0.0001)$ in $2 \mathrm{VO}$ rats. Correspondingly, lenti-AMO153 successfully reversed the decreased expression of

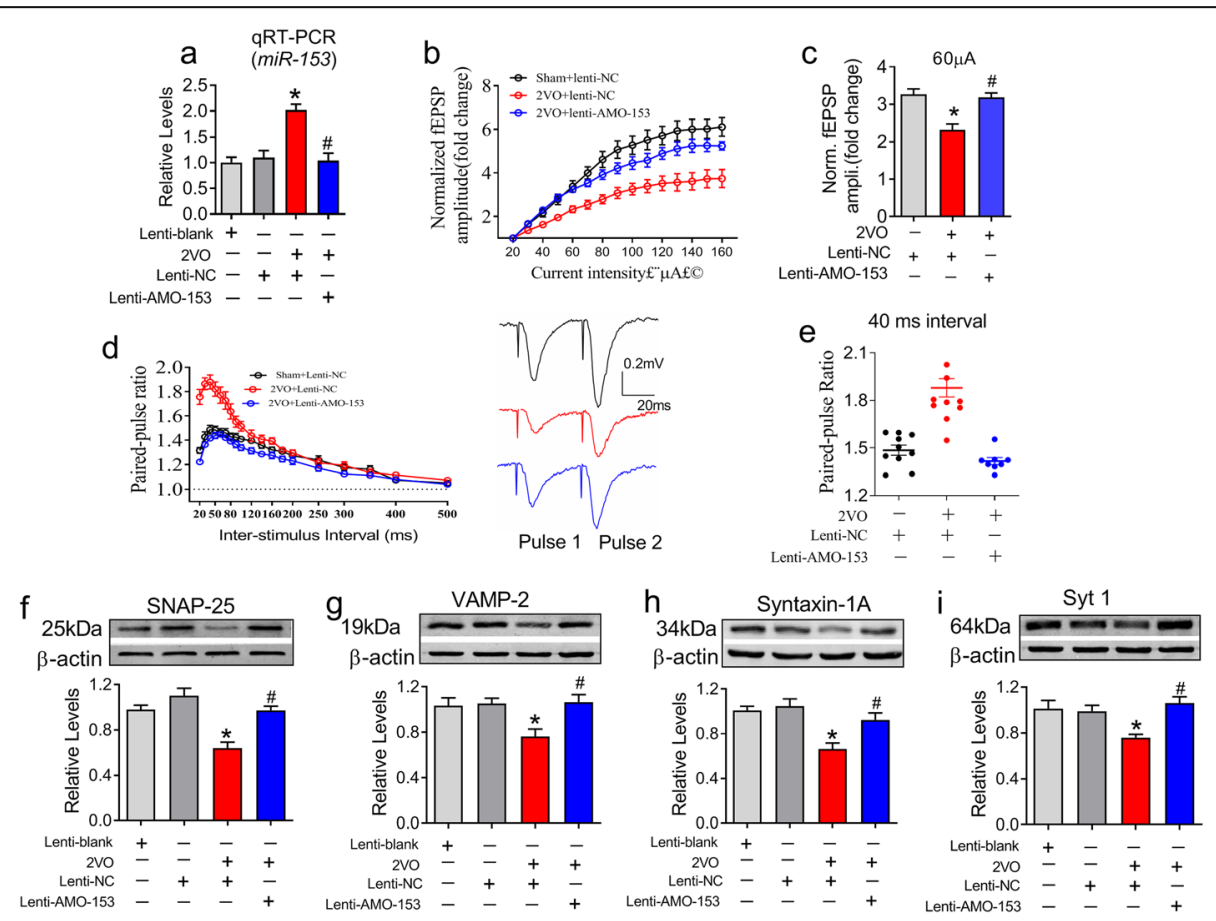

Fig. 6 Knockdown of miR-153 attenuates the decline in hippocampal presynaptic plasticity in 2VO rats. a Lenti-AMO-153 injection inhibited miR153 expression in the hippocampi of 2 VO rats $\left(P_{\text {Levene }}=0.58\right.$, one-way ANOVA: $\left.F=10.301, P=0.0001\right) . \mathbf{n}=6 . \mathbf{b}$ Lenti-AMO-153 attenuated the repression of fEPSP recorded in $2 \mathrm{VO}$ rats. $X^{2}$ Mauchly $=549.258, P<0.0001 ; F_{\text {total }}(14,309)=11.229, P<0.0001$. $\mathbf{c}$ Bar graph showing the fold changes in the fEPSP amplitude under $60 \mu \mathrm{A}$ current stimulation ( $P_{\text {Levene }}=0.598$, one-way ANOVA: $\left.F=8.114, P=0.002\right) . n=10$. $\mathbf{d}$ Lenti-AMO-153 reversed the increase in the PPR in 2 VO rats. Right insertion: sample of PPR fEPSP traces. $X^{2}$ Mauchly $=596.166, P<0.0001 ; F_{(18,505)}=20.561, P<0.0001$. $n=10$ for the 2VO group, $n=9$ for the lenti-NCgroup, $n=8$ for the lenti-AMO-153 group. e Lenti-AMO-153 prevented the increase in the PPR in 2VO rats $\left(P_{\text {Levene }}=0.001\right.$, one-way ANOVA: $\left.F=31.707, P<0.0001\right)$. $\mathbf{f}-\mathbf{i}$ Lenti-AMO-153 derepressed the protein levels of SNAP-25 $\left(P_{\text {Levene }}=0.324\right.$, one-way ANOVA: $F=15.658, P<0.0001), V A M P-2\left(P_{\text {Levene }}=0.752\right.$, one-way ANOVA: $\left.F=5.288, P=0.01\right)$, syntaxin- $1 \mathrm{~A}\left(P_{\text {Levene }}=0.817\right.$, one-way ANOVA: $F=$ $9.248, P<0.0001)$ and Syt1 ( $P_{\text {Levene }}=0.246$, one-way ANOVA: $\left.F=6.3 .60, P=0.003\right)$ in hippocampal synaptosomes in 2 VO rats. Fisher's PLSD test was used for the post hoc analyses of the two-group comparisons. $n=6,{ }^{*} P<0.05$ vs Lenti-NC; ${ }^{\#} P<0.05$ vs 2 VO 
SNAP-25 $(P<0.0001)$, VAMP-2 $(P=0.01)$, syntaxin-1A $(P<0.0001)$ and Syt1 $(P=0.003)$ in the hippocampi of $2 \mathrm{VO}$ rats (Fig. $6 \mathrm{f}-\mathrm{i}$ ). These results suggest that lentiAMO-153 reversed not only the release probability but also the expression of vesicle release-related proteins in $\mathrm{CBH}$ rats.

\section{Knockdown of miR-153 attenuates cognitive decline of $2 \mathrm{VO}$ rats}

Subsequently, we investigated whether miR-153 knockdown could prevent the dementia phenotype induced by $2 \mathrm{VO}$. As expected, compared with age-matched $2 \mathrm{VO}$ rats that were transfected with lenti-NC, $2 \mathrm{VO}$ rats transfected with lenti-AMO-153 exhibited a significantly shortened latency to arrive at the platform (Fig. 7a, $P=$ $0.001)$. In the probe trial, lenti-AMO-153 treatment increased the number of platform crossings in $2 \mathrm{VO}$ rats (Fig. $7 \mathrm{~b}$ and c, $P=0.08$ ). These results imply that knockdown of miR-153 improved spatial memory in $2 \mathrm{VO}$ rats.

\section{Discussion}

We are the first to report that $\mathrm{CBH}$ impairs presynaptic plasticity by disturbing synaptic vesicle release. The disturbed process was due to a blockade of presynaptic vesicle fusion with the presynaptic membrane controlled by $m i R-153$, which posttranscriptionally repressed the expression of fusion-related proteins, including the
SNARE-complex proteins SNAP-25, VAMP-2 and syntaxin-1A and the $\mathrm{Ca}^{2+}$ sensor protein Syt1 by targeting the 3'UTRs of the Snap25, VAMP2, Stx1a and Syt1 genes (Fig. S4). This mechanism not only deepens our understanding of $\mathrm{CBH}$-induced brain dysfunction, but also leads to a new preventive or treatment strategy for $\mathrm{AD}$ or $\mathrm{VaD}$.

The number of formed SNARE complexes is widely known to be the rate-limiting index controlling the quantity and speed of presynaptic vesicle release. Although $2 \sim 3$ SNARE-complexes are sufficient to mediate the fusion process between vesicles and presynaptic membranes, more SNARE complexes trigger greater vesicle release [38]. Here, CBH downregulated the expression of SNAP-25, VAMP-2 and syntaxin-1A, suggesting that $\mathrm{CBH}$ induced vesicle release impairment may reduce the number of SNARE complexes. Synaptic vesicle fusion is not only mediated by SNARE proteins but also co-regulated by SM proteins that control fast vesicle release by opening the fusion-pore by engaging in the formation of the trans-SNARE/SM-complex [15, 39]. However, in our study, Munc-18-1 expression was unchanged in the hippocampi of $2 \mathrm{VO}$ rats, suggesting that the CBH-induced impairment in synaptic fusion is associated with reduced SNARE protein expression but not SM protein expression. Furthermore, prior to fusionpore opening, synaptic vesicles need to be docked and

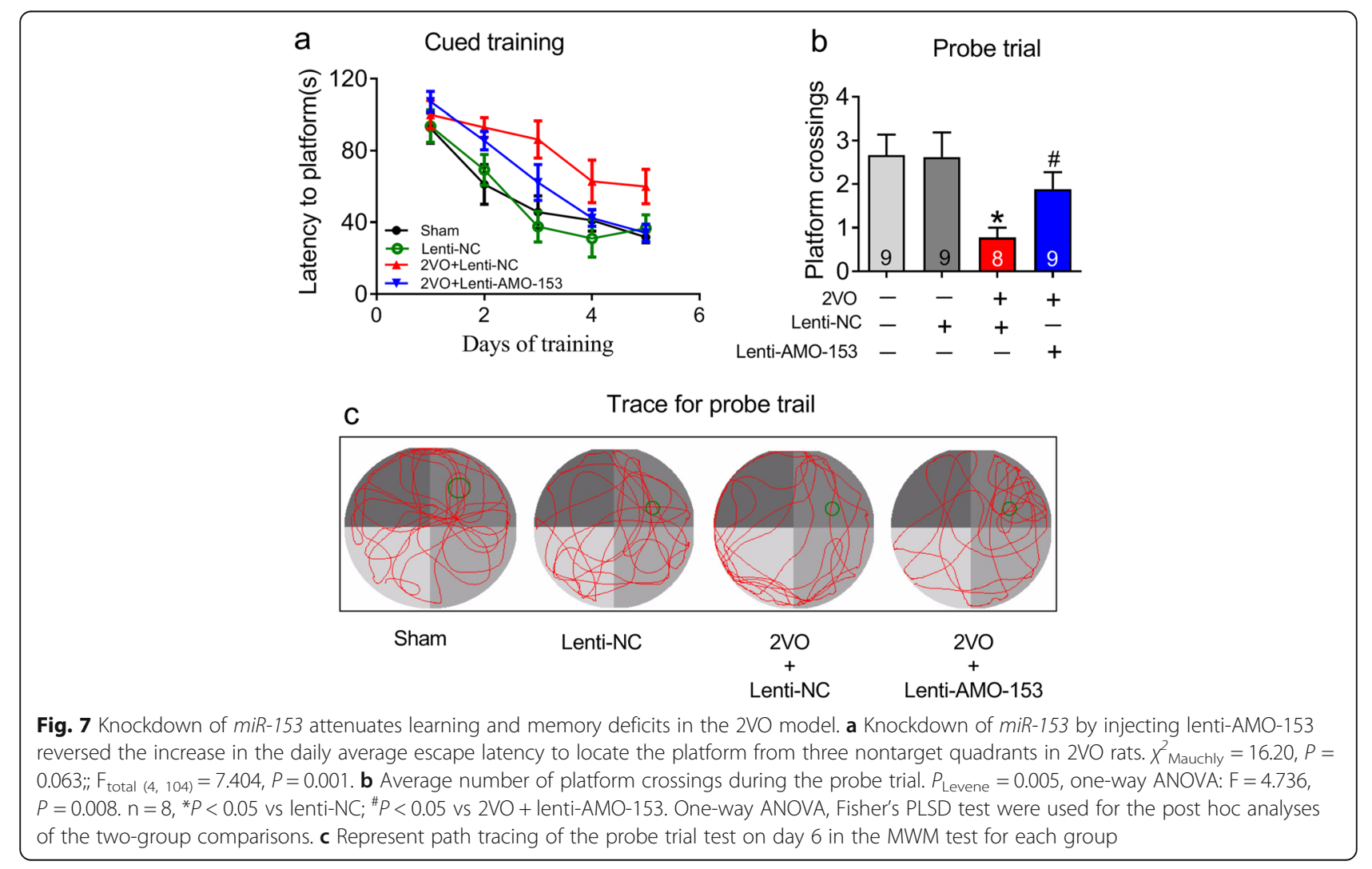


primed by triggering the formation of the trans-SNARE/ SM complex, which is mediated by rapid $\mathrm{Ca}^{2+}$ binding to the $\mathrm{Ca}^{2+}$-sensor protein Syt1 [40,41]. Interestingly, the protein level of Syt1 was significantly reduced in the rat hippocampi following $\mathrm{CBH}$; however, the level of its co-factor complexin $1 / 2$ was unchanged. Thus, the above data provide profound evidence that $\mathrm{CBH}$ impairs presynaptic plasticity through effects on vesicle fusionrelated proteins.

A previous study reported that $m i R-153$ is a contextual fear-induced miRNA and affects fear memory by targeting the Snap 25 and Vamp2 genes [23]. Here, we first reported that $m i R-153$ is also a CBH-induced miRNA. In addition, we found that increased miR-153 levels in the plasma of $\mathrm{VaD}$ patients, suggesting that $m i R-153$ is a potential biomarker for the preclinical prediction of $\mathrm{VaD}$.

Surprisingly, bioinformatics analysis, siRNA and miRmasking experiments revealed that all the vesicle fusionrelated proteins with altered expression in the hippocampi of $\mathrm{CBH}$ rats, including SNAP-25, VAMP-2, syntaxin-1A and Syt1, are miR-153 targets. Notably, the mRNA level of Snap 25 was significantly increased in the hippocampi of $\mathrm{CBH}$ rats, whereas the mRNA level of VAMP2, Stx1a and Syt1 remained unchanged; however, the protein level of SNAP-25 was decreased in the same with manner as that VAMP-2, syntaxin-1A and Syt1. This phenomenon suggests that the inhibitory effect of miR-153 on SNAP-25 was the strongest among these proteins, which was further proven by the luciferase reporter gene expression assay. To clarify the involvement of miR-153 in the CBH-induced presynaptic vesicle release impairment, we performed both in vitro and in vivo experiments. First, using the FM1-43 staining method, we demonstrated that miR-153 gain-of-function markedly blocked $\mathrm{KCl}$-induced vesicle release in NRNs, and this effect of miR-153 was completely prevented by the cotransfection of the antisense AMO-153. Subsequently, by performing four gene-specific miR-masks, we found that the contributions of these four proteins to the miR-153-induced impairment in vesicle release were different. For example, Vamp2-ODN and Snap25-ODN almost completely blocked the miR-153-induced reduction in vesicle release within $5 \mathrm{~s}$ after $\mathrm{KCl}$ stimulation, having the same effect of all four ODNs combined. At $10 \mathrm{~s}$ after $\mathrm{KCl}$ application, the degree of the rescue effect (from strong to weak) of each individual ODN was Vamp2-ODN > Snap25-ODN > Syt1-ODN > Stx1a-ODN. Interestingly, as time extended to $40 \mathrm{~s}$ after $\mathrm{KCl}$ stimulation, the effect of Stx1a-ODN and Syt1-ODN gradually improved and reached to a level similar to that of Vamp2-ODN. These results indicate that VAMP-2 and/ or SNAP-25 are the rate-limiting proteins under $\mathrm{CBH}$ status. Second, as predicted, in vivo, application of lentipre-miR-153 decreased fEPSP amplitude and increased the PPR in the CA3-CA1 pathway and reduced the expression of the vesicle-related proteins similar to the observed effects of $2 \mathrm{VO}$ surgery. Importantly, all these changes were prevented by lenti-AMO-153 treatment. Hence, these data provide pronounced evidence that miR-153 is involved in the impaired vesicle release process in $2 \mathrm{VO}$ rats by posttranscriptionally regulating the expression of a battery of vesicle-related proteins.

Previous studies have reported that $\mathrm{CBH}$ induces a series of pathological changes involving $A \beta$ aggregation, Tau hyperphosphorylation, dendritic remodelling and cell death through the single microRNA- miR-195, which targets a set of genes associated with the $A \beta$ cascade response, including $A P P$ (encoding amyloid precursor protein, APP) [3], BACE1 (encoding $\beta$ secretases, BACE1) [3], cdk5r1 (encoding p35) [4], Ppme1 (encoding methylesterase 1, PME-1) [42] and tnfrsf21 (encoding death receptor 6, DR6) [5]. Importantly, application of $m i R-195$ by stereotaxic injection of lenti-pre-miR-195 into the hippocampus could partially reduce dementiavulnerability triggered by $2 \mathrm{VO}$ [3].

In this study, we found that the single microRNA- miR153 can regulate a battery of coordinating molecules that control presynaptic vesicle release. Interestingly, knockdown of miR-153 by lenti-AMO-153 also partially attenuated 2VO-induced dementia-vulnerability. These observations suggest that a combination of miR-195 upregulation and miR-153 downregualtion may be the best strategy to prevent or treat cognitive decline induced by $\mathrm{CBH}$.

In the present study, although we demonstrated the direct action of miR-153 on presynaptic vesicle release by its targeting of multiple vesicle release-related proteins, we did not investigate the mechanism by which $\mathrm{CBH}$ upregulates of $m i R-153$. A previous study reported that hypoxia-stimulated endoplasmic reticulum stress promotes $m i R-153$ transcription by activating IRE1 $\alpha$ and its downstream transcription factor X-box binding protein 1 (XBP1). XBP1 directly binds to the promoter of the miR-153 host gene PTPRN [43]. Whether XBP1 alone or together with other elements involves in $\mathrm{CBH}$ induced upregulation of miR-153 needs to be identified further.

\section{Conclusion}

Overexpression of miR-153 controls CBH induced presynaptic vesicle release impairment by posttranscriptionally regulating the expression of four vesicle release-related proteins through targeting the 3'UTRs of the Stx1a, Snap25, Vamp2 and Syt1 genes (Fig. S4). These findings provide new insights into the molecular mechanism of $\mathrm{CBH}$-induced presynaptic plasticity impairment at the miRNA level. Our findings suggest that miR-153 might be a candidate for dementia-targeting gene-therapy in the future. 


\section{Supplementary information}

Supplementary information accompanies this paper at https://doi.org/10. 1186/s12964-020-00551-8.

\section{Additional file 1.}

\section{Abbreviations}

AD: Alzheimer's disease; VaD: vascular dementia; $\mathrm{CBH}$ : chronic brain hypoperfusion; 2VO: bilateral common carotid artery ligation; fEPSPs: field excitatory postsynaptic potentials; PPF: paired-pulse facilitation; SNAREs: soluble N-ethylmaleimide-sensitive factor attachment receptor proteins; VAMP-2: vesicle associated membrane protein 2; SNAP25: synaptosomal-associated protein 25; Syt1: synaptotagmin-1: NRNs: neonatal rat hippocampal and cortical neurons; ODNs: oligodeoxynucleotides; MMSE: Mini-Mental State Examination; MoCA: Montreal Cognitive Assessment; HAMD: Hamilton Depression Scale; TTC: 2,3,5-triphenyltetrazolium chloride; MWM: morris water maze; PPR: paired-pulse ratio; CBF: cerebral blood flow; PBS: phosphate-buffered saline; ISI: interstimulus intervals

\section{Authors' contributions}

J.A designed and supervised the study. J.A., M.L.Y and S.Z. designed the experiments and wrote the manuscript. J.A. and L.H.S. initiated the study and contributed to the preliminary data. S.Z., L.Y., S.Y.H and X.B.A performed electrophysiological experiments. M.L.Y., H.M.Z, S.N.X, and M.J.D performed FM1-43 experiments. M.L.Y., H.M.Z and M. M performed vector cell culture and PCR experiments. M.L.Y., S.N.X, J. Z, H.M.Z., Y. X., Y. Q. and X.Y. Z. performed western blot. M.L.Y., S.N.X. performed virus brain injection, C.M. helped write the manuscript. All authors read and approved the final manuscript.

\section{Funding}

This work was supported by the National Natural Science Foundation of China $(81671052,81870849$ and 81471115) and the Key Project of the Natural Science Foundation of Heilongjiang Province (ZD2018004) to J. A.

\section{Availability of data and materials}

The data used in this study are available from the corresponding author upon reasonable request.

\section{Ethics approval and consent to participate}

Not applicable.

\section{Consent for publication}

Not applicable.

\section{Competing interests}

The authors declare that they have no competing interests.

Received: 1 October 2019 Accepted: 11 March 2020

Published online: 06 April 2020

\section{References}

1. de la Torre J. The vascular hypothesis of Alzheimer's disease: a key to preclinical prediction of dementia using neuroimaging. J Alzheimers Dis. 2018;63:35-52

2. Duncombe J, Kitamura A, Hase Y, Ihara M, Kalaria RN, Horsburgh K. Chronic cerebral hypoperfusion: a key mechanism leading to vascular cognitive impairment and dementia. Closing the translational gap between rodent models and human vascular cognitive impairment and dementia. Clin Sci (Lond). 2017;131:2451-68.

3. Ai J, Sun LH, Che H, Zhang R, Zhang TZ, Wu WC, Su XL, Chen X, Yang G, Li $\mathrm{K}$, et al. MicroRNA-195 protects against dementia induced by chronic brain hypoperfusion via its anti-amyloidogenic effect in rats. J Neurosci. 2013;33: 3989-4001.

4. Sun LH, Ban T, Liu CD, Chen QX, Wang X, Yan ML, Hu XL, Su XL, Bao YN, Sun LL, et al. Activation of Cdk5/p25 and tau phosphorylation following chronic brain hypoperfusion in rats involves microRNA-195 downregulation. J Neurochem. 2015;134:1139-51.
5. Chen X, Jiang XM, Zhao $\sqcup$, Sun LL, Yan ML, Tian Y, Zhang S, Duan MJ, Zhao HM, Li WR, et al. MicroRNA-195 prevents dendritic degeneration and neuron death in rats following chronic brain hypoperfusion. Cell Death Dis. 2017:8:e2850.

6. Che H, Yan Y, Kang XH, Guo F, Yan ML, Liu HL, Hou X, Liu T, Zong DK, Sun $\mathrm{LL}$, et al. MicroRNA-27a promotes inefficient Lysosomal clearance in the hippocampi of rats following chronic brain Hypoperfusion. Mol Neurobiol. 2017;54:2595-610.

7. Scheff SW, Neltner JH, Nelson PT. Is synaptic loss a unique hallmark of Alzheimer's disease? Biochem Pharmacol. 2014;88:517-28.

8. Selkoe DJ. Alzheimer's disease is a synaptic failure. Science. 2002;298:789-91.

9. Chakroborty S, Hill ES, Christian DT, Helfrich R, Riley S, Schneider C, Kapecki N, Mustaly-Kalimi S, Seiler FA, Peterson DA, et al. Reduced presynaptic vesicle stores mediate cellular and network plasticity defects in an earlystage mouse model of Alzheimer's disease. Mol Neurodegener. 2019:14:7.

10. Yao ZH, Yao XL, Zhang SF, Hu JC, Zhang Y. Tripchlorolide may improve spatial cognition dysfunction and synaptic plasticity after chronic cerebral Hypoperfusion. Neural Plast. 2019;2019:2158285.

11. Ueno Y, Koike M, Shimada Y, Shimura H, Hira K, Tanaka R, Uchiyama Y, Hattori N, Urabe T. L-carnitine enhances axonal plasticity and improves white-matter lesions after chronic hypoperfusion in rat brain. J Cereb Blood Flow Metab. 2015;35:382-91.

12. Sudhof TC. A molecular machine for neurotransmitter release: synaptotagmin and beyond. Nat Med. 2013;19:1227-31.

13. Zhou P, Bacaj T, Yang X, Pang ZP, Sudhof TC. Lipid-anchored SNAREs lacking transmembrane regions fully support membrane fusion during neurotransmitter release. Neuron. 2013;80:470-83.

14. Zhou P, Pang ZP, Yang X, Zhang Y, Rosenmund C, Bacaj T, Sudhof TC. Syntaxin-1 N-peptide and Habc-domain perform distinct essential functions in synaptic vesicle fusion. EMBO J. 2013;32:159-71.

15. Sudhof TC. Neurotransmitter release: the last millisecond in the life of a synaptic vesicle. Neuron. 2013;80:675-90.

16. Greber S, Lubec G, Cairns N, Fountoulakis M. Decreased levels of synaptosomal associated protein 25 in the brain of patients with Down syndrome and Alzheimer's disease. Electrophoresis. 1999;20:928-34.

17. Shimohama S, Kamiya S, Taniguchi T, Akagawa K, Kimura J. Differential involvement of synaptic vesicle and presynaptic plasma membrane proteins in Alzheimer's disease. Biochem Biophys Res Commun. 1997:236:239-42.

18. Scheff SW, Price DA, Ansari MA, Roberts KN, Schmitt FA, Ikonomovic MD, Mufson EJ. Synaptic change in the posterior cingulate gyrus in the progression of Alzheimer's disease. J Alzheimers Dis. 2015;43:1073-90.

19. Sinclair LI, Tayler HM, Love S. Synaptic protein levels altered in vascular dementia. Neuropathol Appl Neurobiol. 2015;41:533-43.

20. Siegert S, Seo J, Kwon EJ, Rudenko A, Cho S, Wang W, Flood Z, Martorell AJ, Ericsson M, Mungenast AE, Tsai LH. The schizophrenia risk gene product miR-137 alters presynaptic plasticity. Nat Neurosci. 2015;18:1008-16.

21. Hu S, Wang H, Chen K, Cheng P, Gao S, Liu J, Li X, Sun X. MicroRNA-34C Downregulation ameliorates amyloid-beta-induced synaptic failure and memory deficits by targeting VAMP2. J Alzheimers Dis. 2015;48:673-86.

22. Mannironi C, Biundo A, Rajendran S, De Vito F, Saba L, Caioli S, Zona C, Ciotti T, Caristi S, Perlas E, et al. miR-135a regulates synaptic transmission and anxiety-like behavior in amygdala. Mol Neurobiol. 2018;55:3301-15.

23. Mathew RS, Tatarakis A, Rudenko A, Johnson-Venkatesh EM, Yang YJ, Murphy EA, Todd TP, Schepers ST, Siuti N, Martorell AJ, et al. A microRNA negative feedback loop downregulates vesicle transport and inhibits fear memory. Elife. 2016;5.

24. Wei C, Thatcher EJ, Olena AF, Cha DJ, Perdigoto AL, Marshall AF, Carter BD, Broadie K, Patton JG. miR-153 regulates SNAP-25, synaptic transmission, and neuronal development. PLoS One. 2013:8:e57080.

25. Peng Y, Xu S, Chen G, Wang L, Feng Y, Wang X. I-3-n-Butylphthalide improves cognitive impairment induced by chronic cerebral hypoperfusion in rats. J Pharmacol Exp Ther. 2007:321:902-10.

26. Peng $Y$, Sun J, Hon S, Nylander AN, Xia W, Feng Y, Wang X, Lemere CA. L-3n-butylphthalide improves cognitive impairment and reduces amyloid-beta in a transgenic model of Alzheimer's disease. J Neurosci. 2010;30:8180-9.

27. Sun LL, Duan MJ, Ma JC, Xu L, Mao M, Biddyut D, Wang Q, Yang C, Zhang S, $\mathrm{Xu} Y$, et al. Myocardial infarction-induced hippocampal microtubule damage by cardiac originating microRNA-1 in mice. J Mol Cell Cardiol. 2018;120:12-27.

28. Ting JT, Daigle TL, Chen Q, Feng G. Acute brain slice methods for adult and aging animals: application of targeted patch clamp analysis and optogenetics. Methods Mol Biol. 2014;1183:221-42. 
29. Mathis DM, Furman JL, Norris CM. Preparation of acute hippocampal slices from rats and transgenic mice for the study of synaptic alterations during aging and amyloid pathology. J Vis Exp. 2011.

30. Villanueva-Castillo C, Tecuatl C, Herrera-Lopez G, Galvan EJ. Aging-related impairments of hippocampal mossy fibers synapses on CA3 pyramidal cells. Neurobiol Aging. 2017:49:119-37.

31. Kamat PK, Kalani A, Tyagi N. Method and validation of synaptosomal preparation for isolation of synaptic membrane proteins from rat brain. MethodsX. 2014;1:102-7.

32. Gaffield MA, Betz WJ. Imaging synaptic vesicle exocytosis and endocytosis with FM dyes. Nat Protoc. 2006;1:2916-21.

33. Ivannikov MV, Sugimori M, Llinas RR. Synaptic vesicle exocytosis in hippocampal synaptosomes correlates directly with total mitochondrial volume. J Mol Neurosci. 2013;49:223-30.

34. Sun LH, Yan ML, Hu XL, Peng LW, Che H, Bao YN, Guo F, Liu T, Chen X, Zhang R, et al. MicroRNA-9 induces defective trafficking of Nav1.1 and Nav1. 2 by targeting Navbeta2 protein coding region in rat with chronic brain hypoperfusion. Mol Neurodegener. 2015;10:36.

35. Ai J, Zhang R, Li Y, Pu J, Lu Y, Jiao J, Li K, Yu B, Li Z, Wang R, et al. Circulating microRNA-1 as a potential novel biomarker for acute myocardial infarction. Biochem Biophys Res Commun. 2010;391:73-7.

36. Farkas E, Luiten PG, Bari F. Permanent, bilateral common carotid artery occlusion in the rat: a model for chronic cerebral hypoperfusion-related neurodegenerative diseases. Brain Res Rev. 2007:54:162-80.

37. Yoon TY, Kweon DH, Shin YK. Chasing the trails of SNAREs and lipids along the membrane fusion pathway. Curr Top Membr. 2011;68:161-84.

38. Gengler S, Hamilton A, Holscher C. Synaptic plasticity in the hippocampus of a APP/PS1 mouse model of Alzheimer's disease is impaired in old but not young mice. PLoS One. 2010;5:e9764.

39. Dulubova I, Khvotchev M, Liu S, Huryeva I, Sudhof TC, Rizo J. Munc18-1 binds directly to the neuronal SNARE complex. Proc Natl Acad Sci U S A 2007;104:2697-702.

40. McMahon HT, Missler M, Li C, Sudhof TC. Complexins: cytosolic proteins that regulate SNAP receptor function. Cell. 1995;83:111-9.

41. Zhou Q, Zhou P, Wang AL, Wu D, Zhao M, Sudhof TC, Brunger AT. The primed SNARE-complexin-synaptotagmin complex for neuronal exocytosis. Nature. 2017;548:420-5.

42. Liu CD, Wang Q, Zong DK, Pei SC, Yan Y, Yan ML, Sun LL, Hao YY, Mao M, Xing WJ, et al. Knockdown of microRNA-195 contributes to protein phosphatase-2A inactivation in rats with chronic brain hypoperfusion. Neurobiol Aging. 2016;45:76-87.

43. Liang H, Xiao J, Zhou Z, Wu J, Ge F, Li Z, Zhang H, Sun J, Li F, Liu R, Chen C. Hypoxia induces miR-153 through the IRE1alpha-XBP1 pathway to fine tune the HIF1alpha/NEGFA axis in breast cancer angiogenesis. Oncogene. 2018; 37:1961-75

\section{Publisher's Note}

Springer Nature remains neutral with regard to jurisdictional claims in published maps and institutional affiliations.

Ready to submit your research? Choose BMC and benefit from:

- fast, convenient online submission

- thorough peer review by experienced researchers in your field

- rapid publication on acceptance

- support for research data, including large and complex data types

- gold Open Access which fosters wider collaboration and increased citations

- maximum visibility for your research: over $100 \mathrm{M}$ website views per year

At $\mathrm{BMC}$, research is always in progress.

Learn more biomedcentral.com/submissions 